\title{
Designing the Undersea Internet of Things (IoT) and Machine-to-Machine (M2M) Communications Using UnderWater Acoustic MIMO Networks
}

\author{
Athanasios G. Lazaropoulos* \\ School of Electrical and Computer Engineering, National Technical University of Athens (NTUA), \\ 9 Iroon Polytechniou Street, Zografou, Athens, Greece 15780
}

Received December 28, 2015; Accepted January 27, 2016; Published January 29, 2016

This review paper tries to assess the spectral-efficient (SE) and energy-efficient (EE) performance of underwater acoustic multiple-input multiple-output (UWA/MIMO) networks. Since UWA/MIMO networks define the cutting-edge communications platform of the future's undersea loT and M2M networks, the factors that influence their SE and EE performance are thoroughly examined in this paper.

The contribution of this paper is three-fold. First, the performance of UWA/MIMO networks is studied with regard to appropriate transmission, SE and EE metrics. The SE and EE performance of these networks drastically depends on the used frequency band, the transmitted power, the MIMO scheme properties, the power consumption profile of the deployed UWA system equipment and the topological characteristics of MIMO configurations. In order to achieve the transition from traditional UWA single-input single-output (UWA/SISO) networks to UWA/MIMO networks, a new singular value decomposition MIMO (SVD/MIMO) module, which also permits the theoretical computation of the aforementioned transmission, SE and EE metrics in UWA networks, is first presented. Second, based on the aforementioned transmission, SE and EE metrics, a SE/EE trade-off relation is proposed in order to investigate the combined SE and EE performance of UWA/MIMO networks. On the basis of this SE/EE trade-off relation, it is first revealed that today's UWA system equipment cannot support the further IoT broadband exploitation with satisfactory EE performance. Third, the concepts of multi-hop UWA communications and standard UWA topologies are outlined and promoted so that further SE and EE improvement can concurrently occur. These concepts are quantitatively validated by the SE and EE metrics as well as the SE/EE trade-off curves.

Based on the findings of this paper, suitable transmitted power levels and better design of UWA/MIMO configurations are promoted so that: (i) SE and EE requirements can be satisfied at will; and (ii) EE-oriented high-bitrate M2M communications network design can be established.

Keywords: Internet of Things (IoT), Machine-to-Machine (M2M), UnderWater Acoustic (UWA) channel modeling, statistical performance metrics, spectral-efficient (SE) metrics, energy-efficient (EE) metrics, multi-input multi-output (MIMO) networks. 


\section{I: Introduction}

Nowadays, the analysis and design of either Internet of Things (IoT) or Machine-to-Machine (M2M) networks or underwater acoustic (UWA) communications networks are receiving an increased interest by both researchers and practitioners due to the plethora of supported civil and commercial applications. Actually, their integration may open new horizons in communication among divers and underwater vehicles, remote control in off-shore industries, pollution monitoring, discovery of new resources, tactical surveillance of underwater objects as well as scientific exploration of the oceans [1]-[5]. In fact, UWA networks can become the key to delivering IoT and M2M facilities in remote off-shore and underwater areas through the exploitation of their spectral-efficient (SE) potential. At the same time, the development of an advanced integrated IP-based system via UWA technology in IoT and M2M framework may offer new useful applications like support for underwater robots, sonar system improvements, aircraft black box detection, backbone for dense underwater sensor networks and real-time seismic monitoring.

Meanwhile, energy efficiency in communications networks becomes a growing concern. Communications providers focus on maintaining and increasing their profitability by reducing their power consumption. This fervent interest of communications providers towards the reduction of the carbon footprint of their communications networks motivates the continuous exploration of technologies in order to achieve higher energy-efficient (EE) performances [6].

To achieve higher SE and EE performances in UWA networks, the allocation of the appropriate resources such as power and bandwidth as well as the appropriate design of signals and processing algorithms on UWA network layers demand accurate UWA channel models. However, the channel modeling of UWA networks is afflicted by the harshness of the acoustic propagation medium [7]. A UWA channel is characterized by its extremely complex surrounding communications environment that suffers from frequency-dependent path loss, distance-dependent attenuation, multipath propagation, low speed of sound and high noise variations. In addition to the previous problems, the impulse response of UWA channels present time-varying low-pass behavior further aggravated with Doppler shifting and spreading [8], [9]. Therefore, the aforementioned inherent peculiarities of UWA channels combined with the associated underwater deployment difficulties preclude direct application of available wireline and wireless channel modeling techniques, thus, necessitating novel approaches during their analysis [7]. Recently, further complexity during the UWA channel modeling has been added due to the developments regarding multiple-input multiple-output (MIMO) transmission schemes and coexistence of installed UWA networks with other broadband communications networks [10]-[12]. As it is going to be presented in this paper, the deployment of UWA/MIMO networks seems to efficiently mitigate the inherent difficulties of UWA networks improving their SE and EE performance.

Although multi-port UWA networks do not require any additional wiring, four major burdens, which are going to be analyzed in this paper, hinder their further SE and EE performance boost as well as the widespread deployment of IoT and M2M communications networks under the surface of the sea, namely: (i) the limited bandwidth due to the high distance-dependent attenuation; (ii) the intense and fluctuating noise environment; and (iii) the power consumption in relation with the overall UWA network capacity performance. In this paper, the concepts of multi-hop UWA communications 
and standard UWA topologies are highlighted so that further SE and EE improvement can occur in the near future.

However, during the recent efforts to introduce muti-port systems in UWA networks, the key parameter in network design has remained the selection of the optimum number of transmit and receive transducers, which succeeds in maximizing the SE performance. In this paper, the optimization problem is differentiated by taking under consideration both SE and EE performance of single- and multi-port UWA networks. Towards that direction, the SE and EE performance of different single- and multi-port UWA schemes is investigated when: (i) different injected power levels; (ii) different noise conditions; and (iii) different MIMO configuration properties; occur. The results highlight the today's UWA network design dilemma between high SE performance and satisfactory EE operation.

In order to quantify this UWA network design dilemma and define an EE-oriented high-bitrate IoT system design, new SE and EE metrics as well as a new SE/EE trade-off relation is demonstrated. On the basis of the numerical results of the theoretically and experimentally well-validated ray theory of [13]-[17], important transmission metrics -such as the end-to-end channel attenuation-, SE metrics -such as the cumulative capacity and capacity- and EE metrics - such as the total average power consumption, EE cumulative capacity and EE capacity- are reported. Note that ray theory is expanded in this paper with a new singular value decomposition (SVD) module suitable for the UWA/MIMO networks that allows the upgrade of the traditional UWA single-input single-output (SISO) analysis to the UWA/MIMO analysis of this paper. Also, in accordance with recently proposed trade-off relations between capacity performance and power consumption in other communications systems [18], [19], new SE/EE trade-off curves that relate the aforementioned SE and EE metrics are featured when different power consumption scenarios for the UWA/MIMO system equipment occur (e.g. acoustic modems with frequency mixer and frequency synthesizer versus software defined acoustic modems). Further insights, such as how to improve the operation point onto the occurred SE/EE trade-off curves through appropriate combination of different injected power levels and MIMO schemes, are given. Finally, this paper aims at influencing the practical UWA system design towards wider use of IoT concerning: (i) the more SE and EE operation of UWA networks; and (ii) the turn towards more adaptive UWA/MIMO networks.

The rest of the paper is organized as follows: In Section II, the UWA network configurations, which will be used in undersea IoT environment, are presented. Ray theory is also highlighted with the necessary assumptions concerning UWA propagation and transmission. Section III deals with the SVD/MIMO module that allows the transition from the traditional UWA/SISO analysis to the UWA/MIMO one. Section IV summarizes the UWA channel properties that are involved in the following simulation analysis: injected power levels, noise features and UWA/MIMO system parameters related to power consumption. In Section $\mathrm{V}$, a description of the new transmission, SE and EE metrics used in this paper is reported. In Section VI, simulation results and conclusions concerning SE and EE performance of UWA networks are provided, aiming at marking out how a series of factors influence UWA transmission and the corresponding metrics. On the basis of the confirmed trade-off between SE and EE performance, solutions for high-bitrate UWA/MIMO network design that is suitable for the wider use of undersea IoT and M2M communications networks are proposed. Towards that direction, the concepts of multi-hop UWA communications and standard 
UWA topologies are highlighted. In addition, a road map for future research regarding UWA/MIMO networks is presented. Section VII concludes the paper.

\section{The UWA Configurations, Ray Theory and UWA Transfer Function}

The need for the deployment of undersea IoT solutions requires the design of UWA communications systems with improved performance and robustness. This implies accurate and efficient channel models. However, due to the physical nature of the UWA channels, their modeling becomes a challenging issue. Significant inherent deficiencies, such as frequency-dependent attenuation, time-varying multipath propagation, low propagation speed and external noise interferences, degrade the performance of UWA channels [15], [20], [21]. In this Section, the salient characteristics of UWA channel propagation and transmission as well as the ray theory, which offers an accurate deterministic description of UWA/SISO channels, are briefly presented.

\section{A. UWA Configurations}

The UWA configurations differ considerably from transmission via the traditional wireline and wireless communications media. This is due to the significant differences of the network structure and the physical properties of the sea-water, seabed and surface.

A typical configuration that can be used for the analysis of UWA networks is depicted in Fig. 1. $n_{\mathrm{T}}$ transmit transducers are suspended one above the other horizontally and vertically spaced by $\Delta_{\mathrm{zT}}$ and $\Delta_{\mathrm{yT}}$, respectively. The shallowest transmit transducer $\mathrm{T}_{1}$ is horizontally and vertically located at $z_{\mathrm{T} 1}$ and $y_{\mathrm{T} 1}$, respectively, denoted as $\left(z_{\mathrm{T} 1}, y_{\mathrm{T} 1}\right)$, hereafter. Similarly to transmit transducers, $n_{\mathrm{R}}$ receive transducers are

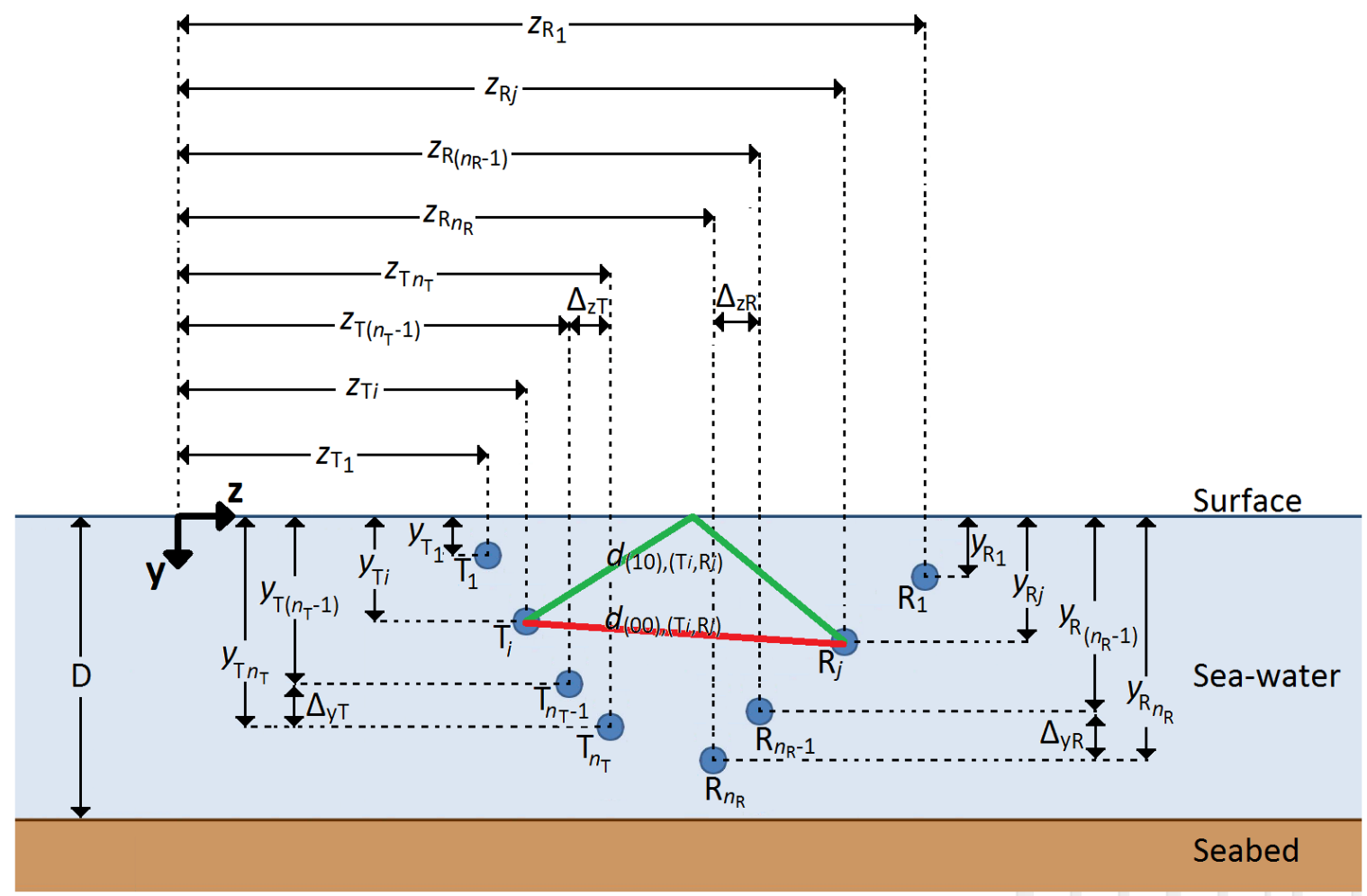

Fig. 1. A typical UWA/MIMO configuration as well as two representative rays. 
deployed one above the other horizontally and vertically spaced by $\Delta_{\mathrm{zR}}$ and $\Delta_{\mathrm{yR}}$, respectively. The shallowest receive transducer $\mathrm{R}_{1}$ is located at $\left(z_{\mathrm{R} 1}, y_{\mathrm{R} 1}\right)$. The water depth $D$ ranges from few meters to $100 \mathrm{~m}$ allowing the assumption of a surrounding shallow water environment [13].

With reference to Fig. 1, the transmit transducer $\mathrm{T}_{i}, i=1, . ., n_{\mathrm{T}}$ and the receive transducer $\mathrm{R}_{j}, j=1, . ., n_{\mathrm{R}}$ are located at $\left(z_{\mathrm{T} i}, y_{\mathrm{T} i}\right), i=1, . ., n_{\mathrm{T}}$ and $\left(z_{\mathrm{R} j}, y_{\mathrm{R} j}\right), j=1, . ., n \mathrm{R}$, respectively, where

$$
\begin{gathered}
z_{\mathrm{T} i}=z_{\mathrm{T} 1}+\left[(i-1) \times \Delta_{\mathrm{zT}}\right], i=1, . ., n \mathrm{~T} \\
y_{\mathrm{T} i}=y_{\mathrm{T} 1}+\left[(i-1) \times \Delta_{\mathrm{yT}}\right\rfloor, i=1, . ., n \mathrm{~T} \\
z_{\mathrm{R} j}=z_{\mathrm{R} 1}+\left[(j-1) \times \Delta_{\mathrm{zR}}\right], j=1, . ., n \mathrm{R} \\
y_{\mathrm{R} j}=y_{\mathrm{R} 1}+\left\lfloor(j-1) \times \Delta_{\mathrm{yR}}\right\rfloor, j=1, . ., n \mathrm{R}
\end{gathered}
$$

Taking into account eqs (1.1)-(1.4), the distance between the transmit transducer $\mathrm{T}_{i}, i=1, . ., n \mathrm{~T}$ and the receive transducer $\mathrm{R}_{j}, j=1, . ., n \mathrm{R}$ is determined by

$$
d_{(00),(\mathrm{Ti}, \mathrm{Rj})}=\sqrt{\left(z_{\mathrm{T} i}-z_{\mathrm{R} i}\right)^{2}+\left(y_{\mathrm{T} i}-y_{\mathrm{R} i}\right)^{2}}, i=1, . ., n \mathrm{~T}, j=1, . ., n_{\mathrm{R}}
$$

Actually, this distance corresponds to the Line of Sight ("LOS") propagation path in UWA channels between the transmit transducer $\mathrm{T}_{i}, \quad i=1, . ., n \mathrm{~T}$ and the receive transducer $\mathrm{R}_{j}, j=1, . ., n \mathrm{R}$.

\section{B. Ray Theory}

A UWA channel may be viewed as a heavy multipath environment, since signal propagation does not take place only across the aforementioned "LOS" path but signal echoes, denoted as rays, originating from points of discontinuities in propagation speed, such as the sea-surface [22]-[24], sea-seabed [25], [26], or other under-sea objects [27], also occur. In order to compute attenuation and multipath fading, the today's UWA channel models vary from applying empirical equations [28], [29] to using more accurate simulation tools and theories [16], [17].

Among the most theoretically and experimentally verified UWA channel models, ray theory and the theory of normal modes provide the required theoretical basis for UWA channel modeling [13]-[17]. In fact, at high frequencies and short- and medium-range communications link distances, ray theory is the suitable UWA channel model since it can accurately determine the behavior of the coarse multipath rays of UWA channels.

Therefore, in accordance with the ray theory of [13], [30], each ray is characterized by four elements, say: (i) its transmit transducer; (ii) its receive transducer; (iii) the number of its surface reflections; and (iv) the number of its seabed reflections. With reference to Fig. 1, the ray distance between the transmit transducer $\mathrm{T}_{i}, i=1, . ., n \mathrm{~T}$ and the receive transducer $\mathrm{R}_{j}, j=1, . ., n_{\mathrm{R}}$ with $s$ surface reflections and $b$ seabed reflections is determined from [13], [30] 


$$
d_{(s b),(\mathrm{T} i, \mathrm{Rj})}=\left\{\begin{array}{l}
\sqrt{\left(z_{\mathrm{T} i}-z_{\mathrm{R} i}\right)^{2}+\left[2 b D+y_{\mathrm{T} i}-(-1)^{s-b} y_{\mathrm{R} i}\right]^{2}}, \text { for } 0 \leq s-b \leq 1 \\
\sqrt{\left(z_{\mathrm{T} i}-z_{\mathrm{R} i}\right)^{2}+\left[2 b D-y_{\mathrm{T} i}+(-1)^{b-s} y_{\mathrm{R} i}\right]^{2}}, \text { for } 0 \leq b-s \leq 1
\end{array}\right\}, i=1, . ., n \mathrm{~T}, j=1, . ., n_{\mathrm{R}}
$$

while the respective incidence angle of the reflected signal is given by [13], [30]

$$
\theta_{(s b),(\mathrm{Ti}, \mathrm{Rj})}=\left\{\begin{array}{l}
\arctan \left[\frac{\left(z_{\mathrm{T} i}-z_{\mathrm{R} i}\right)^{2}}{2 b D+y_{\mathrm{T} i}-(-1)^{s-b} y_{\mathrm{R} i}}\right], \text { for } 0 \leq s-b \leq 1 \\
\arctan \left[\frac{\left(z_{\mathrm{T} i}-z_{\mathrm{R} i}\right)^{2}}{2 b D-y_{\mathrm{T} i}+(-1)^{b-s} y_{\mathrm{R} i}}\right], \text { for } 0 \leq b-s \leq 1
\end{array}\right\}, i=1, . ., n \mathrm{~T}, j=1, . ., n \mathrm{R}
$$

According to the ray theory, rays are assumed to be straight lines in the case of fluid medium with constant propagation speed (isovelocity) [13], [31]. This assumption is also made in this paper.

\section{Ray Attenuation}

In contrast with the propagation through traditional wireless and wireline communications media, UWA propagation is mainly characterized by significant frequency dependent attenuation with slow speed of propagation. For given ray of distance $d_{(s b),(\mathrm{Ti}, \mathrm{Rj})}, i=1, . ., n \mathrm{~T}, j=1, . ., n \mathrm{R}$ and incidence angle $\theta_{(s b),(\mathrm{Ti}, \mathrm{Rj})}, i=1, . ., n \mathrm{~T}, j=1, . ., n_{\mathrm{R}}$, the corresponding ray attenuation can be considered as the sum result of three attenuation mechanisms, namely [7], [13], [32]: (i) The attenuation mechanism due to the spreading losses: This attenuation mechanism expresses the effect of the expansion of the transmitted power over a wide area in the surrounding media, i.e., sea-water, during the signal propagation from the transmit transducer to the receive one. Its value primarily depends on the UWA configuration geometry and is determined from

$$
A_{(s b),(\mathrm{Ti}, \mathrm{Rj})}^{1, \mathrm{~dB}} \equiv A_{(s b),(\mathrm{Ti}, \mathrm{Rj})}^{1, \mathrm{~dB}}\left(d_{(s b),(\mathrm{Ti}, \mathrm{Rj})}\right)=s p \cdot 10 \log \left[d_{(s b),(\mathrm{Ti}, \mathrm{Rj})}\right], i=1, . ., n \mathrm{~T}, j=1, . ., n_{\mathrm{R}}
$$

where $s p$ is the spreading factor describing the nature of UWA propagation. In the case of the practical spreading of UWA channels [13], $s p$ is assumed equal to 1.5; (ii) The attenuation mechanism due to the path loss: It results from the conversion of the transmitted power into heat over the surrounding medium. This attenuation mechanism strongly depends on the operating frequency of the UWA communications link and on numerous other parameters, such as salinity, gauge pressure, temperature, relaxation frequency, etc.. Typically, the attenuation due to the path loss is determined using Thorp's formulae, namely [33], [34]:

$$
A_{(s b),(\mathrm{Ti}, \mathrm{Rj})}^{2, \mathrm{~dB}} \equiv A_{(s b),(\mathrm{Ti}, \mathrm{Rj})}^{2, \mathrm{~dB}}\left(d_{(s b),(\mathrm{Ti}, \mathrm{Rj})}, f\right)=d_{(s b),(\mathrm{Ti}, \mathrm{Rj})} \cdot 10 \log \left[q^{2}(f)\right], i=1, . ., n_{\mathrm{T}}, j=1, . ., n_{\mathrm{R}}
$$

where

$$
10 \log \left[q^{2}(f)\right]=2.49 \times 10^{-7} f^{2}+0.99 \frac{f^{2}}{f^{2}+1.23 \times 10^{4}}+1.48 \times 10^{-4} \frac{f^{2}}{f^{2}+1.522}
$$

is the absorption coefficient in seawater in $\mathrm{dB} / \mathrm{m}$ and $f$ is the operating frequency in $\mathrm{kHz}$; and (iii) The attenuation mechanism due to the reflection loss: In the case of shallow water environment, this attenuation mechanism describes the effect of signal reflections. As it has already been mentioned, the transmitted signal is partially or totally reflected by hitting the sea-surface, sea-bottom or another under-sea object. Therefore, apart from the 
"LOS" path, a great number of different rays allows the transmitted signal to arrive to the receive transducer creating the multipath environment of UWA channels. The attenuation due to the reflection loss of each ray depends on the operating frequency, the propagation speed, the nature of the obstacles encountered across the propagation path, the distance and the incidence angle [13]. Hence, for given ray, the attenuation due to the reflection loss is determined from

where

$$
A_{(s b),(\mathrm{Ti}, \mathrm{Rj})}^{3, \mathrm{~dB}} \equiv A_{(s b),(\mathrm{Ti}, \mathrm{Rj})}^{3, \mathrm{~dB}}\left(d_{(s b),(\mathrm{Ti}, \mathrm{Rj})}, \theta_{(s b),(\mathrm{Ti}, \mathrm{Rj})}\right)=20 \log \left[\Gamma_{(s b),(\mathrm{Ti}, \mathrm{Rj})}\right], i=1, . ., n \mathrm{~T}, j=1, . ., n_{\mathrm{R}}
$$

$$
\begin{gathered}
\Gamma_{(s b),(\mathrm{Ti}, \mathrm{Rj})}=\left[\Gamma_{(s b),(\mathrm{Ti}, \mathrm{Rj})}^{+} \cdot L_{S S}\right]^{s} \cdot\left[\Gamma_{(s b),(\mathrm{Ti}, \mathrm{Rj})}^{-} \cdot L_{S B}\right]^{b}, i=1, . ., n \mathrm{~T}, j=1, . ., n \mathrm{R} \\
\Gamma_{(s b),(\mathrm{Ti}, \mathrm{Rj})}^{+} \cong 1, i=1, . ., n \mathrm{~T}, j=1, . ., n \mathrm{R} \\
\Gamma_{(s b),(\mathrm{Ti}, \mathrm{Rj})}^{-}=\mid \frac{\frac{p_{1}}{p} \cdot \cos \left(\theta_{(s b),(\mathrm{Ti}, \mathrm{Rj})}\right)-\sqrt{\left(\frac{c}{c_{1}}\right)^{2}-\sin ^{2}\left(\theta_{(s b),(\mathrm{Ti}, \mathrm{Rj})}\right)} \mid, i=1, . ., n \mathrm{~T}, j=1, . ., n \mathrm{R}}{\frac{p_{1}}{p} \cdot \cos \left(\theta_{(s b),(\mathrm{Ti}, \mathrm{Rj})}\right)+\sqrt{\left(\frac{c}{c_{1}}\right)^{2}-\sin ^{2}\left(\theta_{(s b),(\mathrm{Ti}, \mathrm{Rj})}\right)} \mid}
\end{gathered}
$$

In eqs. (8)-(11), $\Gamma_{(s b),(\mathrm{Ti}, \mathrm{Rj})}$ is the total reflection loss, $\Gamma_{(s b),(\mathrm{Ti}, \mathrm{Rj})}^{+}$is the attenuation coefficient due to reflection on the surface, $\Gamma_{(s b),(\mathrm{Ti}, \mathrm{Rj})}^{-}$is the attenuation coefficient due to reflection on the seabed, $L_{S S}$ is the constant reflection loss coefficient due to the surface, $L_{S B}$ is the constant reflection loss coefficient due to the seabed, $\rho$ is the density of the sea-water, $\rho_{1}$ is the density of the sea-bed, $c$ is the propagation speed in the sea-water and $c_{1}$ is the propagation speed in the sea-bed.

Taking into consideration the eqs. (6)-(11), the ray attenuation is given by

$$
A_{(s b),(\mathrm{Ti}, \mathrm{Rj})}^{\mathrm{dB}}=A_{(s b),(\mathrm{Ti}, \mathrm{Rj})}^{1, \mathrm{~dB}}+A_{(s b),(\mathrm{Ti}, \mathrm{Rj})}^{2, \mathrm{~dB}}+A_{(s b),(\mathrm{Ti}, \mathrm{Rj})}^{3, \mathrm{~dB}}, i=1, . ., n \mathrm{~T}, j=1, . ., n_{\mathrm{R}}
$$

\section{Ray Transfer Function and UWA Transfer Function}

Based on eq. (12), for the given ray of distance $d_{(s b),(\mathrm{Ti}, \mathrm{Rj})}, i=1, . ., n \mathrm{~T}, j=1, . ., n_{\mathrm{R}}$ and incidence angle $\theta_{(s b),(\mathrm{Ti}, \mathrm{Rj})}, i=1, . ., n \mathrm{~T}, j=1, . ., n_{\mathrm{R}}$, the corresponding transfer function is determined from

$$
H_{(s b),(\mathrm{Ti}, \mathrm{Rj})}\left\{d_{(s b),(\mathrm{Ti}, \mathrm{Rj})}\right\}=10^{\frac{A_{(s b),(\mathrm{T}, \mathrm{R} \mathrm{j})}^{\mathrm{dB}}}{20}} \cdot e^{-j 2 \pi f \cdot \tau_{(s b),(\mathrm{Ti}, \mathrm{Rj})}}, i=1, . ., n \mathrm{~T}, j=1, . ., n_{\mathrm{R}}
$$

where

$$
\tau_{(s b),(\mathrm{Ti}, \mathrm{Rj})}=\frac{d_{(s b),(\mathrm{Ti}, \mathrm{Rj})}}{c}, i=1, . ., n \mathrm{~T}, j=1, . ., n \mathrm{R}
$$

is the arrival time of the given ray.

In accordance with Finite Impulse Response (FIR) filter and ray theory [13], [30], the UWA channel is modeled by taking into account all possible rays of the occurred multipath environment. Therefore, the UWA channel transfer function between the transmit transducer $\mathrm{T}_{i}, i=1, \ldots, n \mathrm{~T}$ and the receive transducer $\mathrm{R}_{j}, j=1, \ldots, n \mathrm{R}$ is given by:

$$
\begin{array}{r}
H_{(\mathrm{Ti}, \mathrm{Rj})}\{f\} \equiv H_{(\mathrm{Ti}, \mathrm{Rj})}\left(d_{(00),(\mathrm{Ti}, \mathrm{Rj})}, f\right)=H_{(00),(\mathrm{Ti}, \mathrm{Rj})}\left\{d_{(00),(\mathrm{Ti}, \mathrm{Rj})}\right\}+\sum_{s=1}^{+\infty} \sum_{b=s-1}^{s} H_{(s b),(\mathrm{Ti}, \mathrm{Rj})}\left\{d_{(s b),(\mathrm{Ti}, \mathrm{Rj})}\right\}+\sum_{b=1}^{+\infty} \sum_{s=b-1}^{b} H_{(s b),(\mathrm{Ti}, \mathrm{Rj})}\left\{d_{(s b),(\mathrm{Ti}, \mathrm{Rj})}\right\} \\
, i=1, . ., n \mathrm{~T}, j=1, . ., n_{\mathrm{R}} \quad(14)
\end{array}
$$


For the practical computations of this paper and according to [13], instead of the infinite number of reflections in the occurred multipath environment, a finite number of reflections on surface and sea-bed is assumed and is equal to $s_{\max }$ and $b_{\max }$, respectively. Then, the respective closed-form expression of the UWA channel transfer function between the transmit transducer $\mathrm{T}_{i}, i=1, . ., n \mathrm{~T}$ and the receive transducer $\mathrm{R}_{j}, j=1, \ldots, n \mathrm{R}$ is determined from:

$$
\begin{gathered}
H_{(\mathrm{Ti}, \mathrm{Rj})}\{f\} \equiv H_{(\mathrm{Ti}, \mathrm{Rj})}\left(d_{(00),(\mathrm{Ti}, \mathrm{Rj})}, f\right)=H_{(00),(\mathrm{Ti}, \mathrm{Rj})}\left\{d_{(00),(\mathrm{Ti}, \mathrm{Rj})}\right\}+\sum_{s=1}^{s_{\max }} \sum_{b=s-1}^{s} H_{(s b),(\mathrm{Ti}, \mathrm{Rj})}\left\{d_{(s b),(\mathrm{Ti}, \mathrm{Rj})}\right\}+\sum_{b=1}^{b_{\max }} \sum_{s=b-1}^{b} H_{(s b),(\mathrm{Ti}, \mathrm{Rj})}\left\{d_{(s b),(\mathrm{Ti}, \mathrm{Rj})}\right\} \\
, i=1, . ., n \mathrm{~T}, j=1, \ldots, n \mathrm{R}
\end{gathered}
$$

In addition, for the rest of this paper, the $n_{\mathrm{T}}$ transmit transducers of the UWA configuration are located at $z_{\mathrm{T} 1}=\ldots=z_{\mathrm{T} n_{\mathrm{T}}}=0$ (i.e., $\Delta_{\mathrm{zT}}=0$ ) whereas the $n_{\mathrm{R}}$ receive transducers are located at $z_{\mathrm{R} 1}=\ldots=z_{\mathrm{R} n_{\mathrm{R}}}=z$ (i.e., $\Delta_{\mathrm{zR}}=0$ ), without harming the generality of the analysis. Anyway, this is the typical case during multi-port UWA configuration deployment [13], [30].

\section{The MIMO Transmission Analysis of UWA Networks: The New SVD/MIMO Module}

Through a matrix approach, the standard ray theory can be extended to the MIMO ray theory that involves more than three transducers. In order to apply MIMO ray theory, which is based on the standard ray theory already presented in Sec.II, FIR filter theory and SVD modal analysis of [18], the spectral relationship of the $n_{\mathrm{T}} \times n_{\mathrm{R}}$ independent transmission channels is modeled by evaluating their channel transfer functions $H_{i j}\{\cdot\}, i=1, . ., n \mathrm{~T}, j=1, . ., n \mathrm{R}$, namely

$$
H_{i j}\{\cdot\} \equiv H_{(\mathrm{Ti}, \mathrm{Rj})}\{\{\}, i=1, . ., n \mathrm{~T}, j=1, . ., n \mathrm{R}
$$

where $H_{i j}\{\cdot\}, i=1, . ., n \mathrm{~T}, j=1, . ., n_{\mathrm{R}}$ is the element in row $i$ of column $j$ of the $n_{\mathrm{T}} \times n_{\mathrm{R}}$ channel transfer function matrix $\mathbf{H}\{\cdot\}$.

Actually, the $n_{\mathrm{T}} \times n_{\mathrm{R}}$ channel transfer function matrix $\mathbf{H}\{\cdot\}$ relating line voltages $\mathbf{V}(z)=\left[\begin{array}{lll}V_{1}(z) & \cdots & V_{n_{\mathrm{R}}}(z)\end{array}\right]^{\mathrm{T}}$ at position $z$ with line voltages $\mathbf{V}(0)=\left[\begin{array}{lll}V_{1}(0) & \cdots & V_{n_{\mathrm{T}}}(0)\end{array}\right]^{\mathrm{T}}$ at position of the transmit transducers (i.e., $z=0)$ is determined from

$$
\mathbf{V}(z)=\mathbf{H}^{\mathrm{T}}\{\mathbf{V}(0)\}
$$

where $[\cdot]^{\mathrm{T}}$ denotes the transpose of a matrix.

Since, in single- and multi-port UWA networks, the number of active transmit and receive transducers may vary from one to $n_{\mathrm{T}}$ and one to $n_{\mathrm{R}}$, respectively, through a similar matrix expression to eq. (17), $\min \left\{n_{\mathrm{T}}, n_{\mathrm{R}}\right\}$ parallel and independent UWA/SISO channels may occur, appropriately decomposing channel transfer function matrix $\mathbf{H}\{\cdot\}$ using the SVD transformation [18], [35]-[40]:

where

$$
\widetilde{\mathbf{H}}^{\mathrm{m}}\{\cdot\}=\widetilde{\mathbf{T}}_{\mathrm{V}}^{\mathrm{H}} \cdot \mathbf{H}^{+}\{\cdot\} \cdot \widetilde{\mathbf{T}}_{\mathrm{I}}
$$

$$
\left.H_{i j}^{+}\{\}\right\}=\left\{\begin{array}{cc}
H_{i j}\{\cdot\} & \text { if }\left(i \in N_{T} \text { and } j \in N_{R}\right) \\
0 & \text { else }
\end{array}, i, j=1, . ., \max \left\{n_{\mathrm{T}}, n_{\mathrm{R}}\right\}\right.
$$


denotes the element of matrix $\mathbf{H}^{+}\{\cdot\}$ in row $i$ of column $j, N_{T}$ and $N_{R}$ are the active transmit and the active receive transducer sets, respectively, and $\max \{x, y\}$ returns the highest value between $x$ and $y$. From eqs. (18) and (19), $\mathbf{H}^{+}\{\cdot\}$ is the $\max \left\{n_{\mathrm{T}}, n_{\mathrm{R}}\right\} \times \max \left\{n_{\mathrm{T}}, n_{\mathrm{R}}\right\}$ extended channel transfer function matrix whose elements $H_{i j}^{+}\{\}, i,. j=1, . ., \max \left\{n_{\mathrm{T}}, n_{\mathrm{R}}\right\}$ are the extended channel transfer functions, $\widetilde{\mathbf{H}}^{\mathrm{m}}\{\cdot\}$ is a diagonal matrix operator whose elements $\widetilde{H}_{i}^{\mathrm{m}}\{\cdot\}, i=1, \ldots, \min \left\{n_{T}, n_{R}\right\}$ are the singular values of $\mathbf{H}^{+}\{\cdot\}$ and, at the same time, the SVD modal transfer functions, $\min \{x, y\}$ returns the smallest value between $x$ and $y,[\cdot]^{\mathrm{H}}$ denotes the Hermitian conjugate of a matrix, and $\widetilde{\mathbf{T}}_{V}$ and $\widetilde{\mathbf{T}}_{I}$ are $\min \left\{n_{T}, n_{R}\right\} \times \min \left\{n_{T}, n_{R}\right\}$ unitary matrices [36], [37]. Combining eqs. (17)-(19), SVD modal transfer function matrix $\widetilde{\mathbf{H}}^{\mathrm{m}}\{$.$\} may be$ determined given channel transfer function matrix $\mathbf{H}\{\cdot\}$. The latter SVD/MIMO module, which additively operates with the ray theory of Sec.II, permits the transition from the UWA/SISO channel analysis to the UWA/MIMO one.

\section{Power Constraints, Noise and Power Consumption of UWA Systems}

During the multi-port UWA configuration implementation, a number of transmit and receive transducer is deployed undersea. In fact, a transducer is a lightweight device that is able to establish high bitrate short-, medium- and long-range UWA communications links [41]. The proper selection of the used transducers in singleand multi-port UWA networks critically defines the SE performance and power consumption of the respective networks. In Fig. 2, the multiple roles of transducer in the block diagram of an end-to-end UWA communications link are featured. In this Section, a comprehensive analysis concerning power constraints, noise and power consumption of UWA system equipment is presented.

\section{A. Power Constraints}

Observing eq. (10), it is evident that the surface acts as a protective layer against EMI emissions of UWA networks. Actually, attenuation coefficient due to reflection on surface $\Gamma^{+}$is relatively small in magnitude since the impedance mismatches between the sea-water and air. In accordance with [13], when the sea is calm, reflection coefficient tends to be equal to the perfect reflection value 1 whereas when the sea surface is rough due to waves, a small loss will be incurred for every surface interaction. Therefore, as it concerns the operation of UWA networks in the examined $0-100 \mathrm{kHz}$ frequency band, relaxed maximum levels can be considered providing the required compliance with all other communications systems.

With regard to power constraints at frequencies below $100 \mathrm{kHz}$, according to the existing literature [7], [13], [20], [41]-[44], power levels $p(f)$ ranging from $-100 \mathrm{dBm} / \mathrm{Hz}$ to $50 \mathrm{dBm} / \mathrm{Hz}$ may constitute typical injected power spectral density mask (IPSDM) limits for UWA networks. Note that the average uniform IPSDM limits in the UWA literature are assumed to be equal to $-25 \mathrm{dBm} / \mathrm{Hz}$ [13], [20], [44].

\section{B. Noise Characteristics}


According to [9], [13], [44]-[46], several important natural sources of ambient noise degrade the performance of UWA networks at frequency bands of interest. Actually, four types of noise are dominant in UWA channels, namely: (i) Turbulence noise; (ii) Shipping noise; (iii) Noise due to waves; and (iv) Thermal noise.

As it regards the mathematical modeling of the aforementioned noise types, to extend the analysis in the $0-100 \mathrm{kHz}$ frequency range, uniform additive white Gaussian noise (AWGN) PSD level among different UWA/MIMO systems is assumed [44], [47]. In detail, to evaluate the capacity of single- and multi-port UWA networks, a uniform AWGN/PSD level $N(f)$ is assumed to be in the range from $-120 \mathrm{dBm} / \mathrm{Hz}$ to $-30 \mathrm{dBm} / \mathrm{Hz}$ simulating a variety of noise environments. Note that the typical uniform AWGN PSD levels in the UWA literature are assumed to be equal to $-83 \mathrm{dBm} / \mathrm{Hz}$ (default noise conditions) [44], [47].

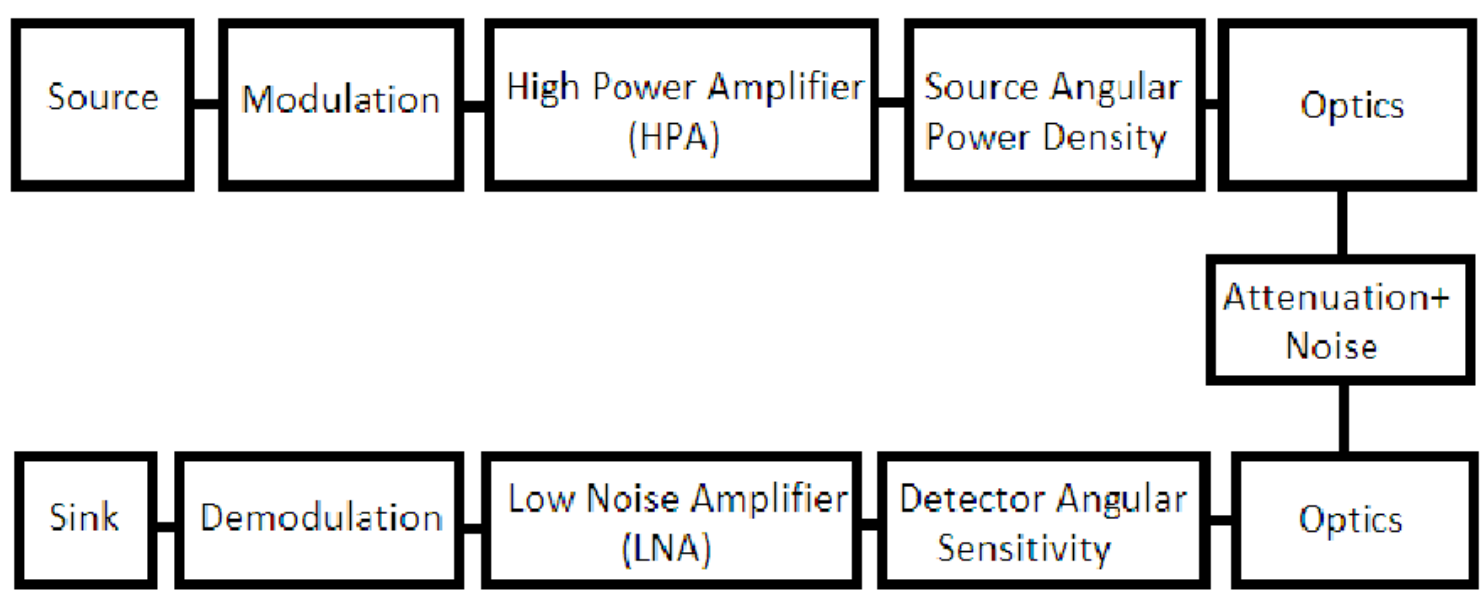

Fig. 2. Block diagram of an end-to-end UWA communications link [41].

\section{Power Consumption}

In accordance with [18] and similarly to other wireless and wireline MIMO communications systems, two types of power consumption are present in UWA transducers [48]-[50]:

- Power Consumption due to Power Amplifiers (Power Consumption Mechanism A). Power amplifiers are the main power consumption blocks in any advanced communications system. Power consumption due to power amplifiers 
mainly depends on the imposed IPSDM limits and the noise [19], [48], [49] and is determined by

$$
P_{\mathrm{PA}} \equiv P_{\mathrm{PA}}(L=K)=\left(\frac{\xi}{n}\right) M_{l} f_{s} \sum_{q=0}^{L-1}\left\{\left[\left\langle N\left(q f_{s}\right)\right\rangle_{\mathrm{Li}}\right]^{-1} \cdot\left\langle N_{r}\left(q f_{s}\right)\right\rangle_{\mathrm{Li}} \cdot\left\langle p\left(q f_{s}\right)\right\rangle_{\mathrm{Li}}\right\}
$$

where $\langle\cdot\rangle_{\mathrm{Li}}$ is an operator that converts $\mathrm{dBm} / \mathrm{Hz}$ into a linear power ratio $(\mathrm{W} / \mathrm{Hz})$, $\xi$ is the power amplifier output backoff [49], $n$ is the drain efficiency [48], [51], $N_{r}(f)$ is the total effective PSD noise in $\mathrm{dBm} / \mathrm{Hz}$ at the receiver input, $M_{l}$ is the link margin compensating the hardware process variations and other additive noise or interference [48], $K$ is the number of subchannels in the UWA signal frequency range of interest and $f_{s}$ is the flat-fading subchannel frequency spacing (details concerning $K$ and $f_{\mathrm{s}}$ are given in Secs. V and VI).

- Power Consumption due to all other Circuit Blocks (Power Consumption Mechanism B). This type of power consumption is related to all other circuit blocks -apart from power amplifiers- of which the single- and multi-port UWA systems consists, namely:

$P_{\mathrm{C}} \equiv P_{\mathrm{C}}(L=K) \cong n_{T}\left(P_{D A C}+P_{m i x}+P_{f i l t}\right)+2 P_{s y n}+n_{R}\left(P_{L N A}+P_{m i x}+P_{I F A}+P_{f i l r}+P_{A D C}\right)$

where $P_{D A C}, P_{m i x}, P_{\text {filt }}, P_{s y n}, P_{L N A}, P_{I F A}, P_{\text {filr }}$ and $P_{A D C}$ are the power consumption values for the Digital-to-Analog Converter, the frequency mixer, the active filters at the transmitting end, the frequency synthesizer, the low-noise amplifier, the intermediate frequency amplifier, the active filters at the receive transducer and the Analog-to-Digital Converter, respectively [48], [49].

Based on eqs. (20) and (21), the total average power consumption $P_{\text {tot }}$ of singleand multi-port UWA systems is given by the sum of the aforementioned two types of power consumption, say:

$$
P_{\text {tot }}(L=K)=P_{\mathrm{PA}}+P_{\mathrm{C}}
$$

During the last years, the rapid development in UWA communications was enhanced by analogous progress in UWA acoustic modems. In fact, the vast majority of the modern UWA acoustic modems are going to be software defined. This implies that there are no frequency mixer and frequency synthesizer in the hardware, in contrast to traditional radio modems. Note that, during the simulation results of Sec. VI, two different power consumption scenarios for the UWA/MIMO systems are going to be examined namely: (i) Acoustic modems with frequency mixer and frequency synthesizer (denoted as power consumption scenario A); and (ii) software defined acoustic modems (denoted as power consumption scenario B).

\section{SE and EE Metrics of Single- and Multi-Port UWA Networks}

In accordance with [18], several useful SE and EE metrics are presented and mathematically defined. In this Section, the performance of single- and multi-port UWA systems is quantitatively evaluated. More specifically, the SE and EE metrics that are applied in order to assess the performance of these UWA systems are:

- The capacity. In information theory, the Shannon-Hartley theorem defines the maximum achievable transmission rate at which information can be reliably transmitted over a UWA communications channel of a specific bandwidth in the 
presence of noise. More specifically, the capacity of the UWA/SISO channel from transmit transducer $\mathrm{T}_{i}, i=1, . ., n \mathrm{~T}, \operatorname{card}\left(N_{T}\right)=1$ to receive transducer $\mathrm{R}_{j}, j=1, . ., n \mathrm{R}, \operatorname{card}\left(N_{R}\right)=1$ is given by [18], [52], [53]

$C_{i j}^{\mathrm{SISO}} \equiv C_{(\mathrm{Ti}, \mathrm{Rj})}^{\mathrm{SISO}}(L=K)=f_{s} \sum_{q=0}^{L-1} \log _{2}\left\{1+\left[S N R\left(q f_{s}\right) \cdot\left|H_{i j}^{+}\left(q f_{s}\right)\right|^{2}\right]\right\}, i=1, . ., n \mathrm{~T}, j=1, . ., n_{\mathrm{R}}$

where

$$
\operatorname{SNR}(f)=\langle p(f)\rangle_{\mathrm{Li}} /\langle N(f)\rangle_{\mathrm{Li}}
$$

is the UWA signal-to-noise ratio (SNR),

$$
K=100 \mathrm{kHz} / f_{s}
$$

is the number of subchannels in the $0-100 \mathrm{kHz}$ frequency range of interest and card(·) returns the cardinality of a set. With reference to eq. (23), the elements $C_{i j}^{\mathrm{SISO}}$ with $i=j$ correspond to SISO co-channel (SISO/CC) UWA systems, while those with $i \neq j$ correspond to the SISO cross-channel (SISO/XC) UWA ones.

Similarly, the capacity of the $1 \times \operatorname{card}\left(N_{R}\right)$ single-input multiple-output (SIMO) systems from the transmit transducer $\mathrm{T}_{i}, i=1, . ., n \mathrm{~T}, \operatorname{card}\left(N_{T}\right)=1$ to receive transducers $\mathrm{R}_{j}, j \in N_{R}, \operatorname{card}\left(N_{R}\right) \geq 2$ is given by

$$
C_{i, N_{R}}^{\mathrm{SIMO}} \equiv C_{(\mathrm{Ti}, \mathrm{Rj}), j \in N_{R}}^{\mathrm{SIMO}}(L=K)=f_{s} \sum_{q=0}^{L-1} \log _{2}\left\{1+\operatorname{SNR}\left(q f_{s}\right) \cdot \sum_{j \in N_{R}}\left|H_{i j}^{+}\left(q f_{s}\right)\right|^{2}\right\}, i=1, . ., n \mathrm{~T}
$$

In the case of $\operatorname{card}\left(N_{T}\right) \times 1$ multiple-input single-output (MISO) systems, their capacity from the transmit transducers $\mathrm{T}_{i}, i \in N_{T}, \operatorname{card}\left(N_{T}\right) \geq 2$ to receive transducer $\mathrm{R}_{j}, j=1, . ., n \mathrm{R}, \operatorname{card}\left(N_{R}\right)=1$ is given by [54]

$$
C_{N_{T}, j}^{\mathrm{MISO}} \equiv C_{(\mathrm{Ti}, \mathrm{Rj}), i \in N_{T}}^{\mathrm{MIO}}(L=K)=f_{s} \sum_{q=0}^{L-1} \log _{2}\left\{1+\frac{S N R\left(q f_{s}\right)}{\operatorname{card}\left(N_{T}\right)} \cdot \sum_{j \in N_{T}}\left|H_{i j}^{+}\left(q f_{s}\right)\right|^{2}\right\}, j=1, . ., n \mathrm{R}
$$

Finally, in the general case of $\operatorname{card}\left(N_{T}\right) \times \operatorname{card}\left(N_{R}\right)$ MIMO systems, their capacity from the transmit transducers $\mathrm{T}_{i}, i \in N_{T}, \operatorname{card}\left(N_{T}\right) \geq 2$ to receive transducers $\mathrm{R}_{j}, j \in N_{R}, \operatorname{card}\left(N_{R}\right) \geq 2$ is given by [35], [37], [39], [54], [55]

$$
C_{N_{T}, N_{R}}^{\mathrm{MIMO}} \equiv C_{(\mathrm{Ti}, \mathrm{Rj}), i \in N_{T}, j \in N_{R}}^{\mathrm{MIMO}}(L=K)=f_{s} \sum_{q=0}^{L-1} \sum_{i=1}^{\min \left\{\operatorname{card}\left(N_{T}\right), \operatorname{card}\left(N_{R}\right)\right\}} \log _{2}\left\{1+\frac{S N R\left(q f_{s}\right)}{\operatorname{card}\left(N_{T}\right)} \cdot\left|\widetilde{H}_{i}^{m}\left(q f_{s}\right)\right|^{2}\right\}
$$

Note that both eqs. (27) and (28) are based on equal power uncorrelated sources as the common case is adopted in this paper.

- The cumulative capacity. In accordance with [18], it is the cumulative upper limit of information (bps) which can be reliably transmitted over an end-to-end UWA channel defining the upper bound of capacity for given IPSDM limits and frequency band. With reference to eqs. (23), (26)-(28) and for given frequency $f$, the cumulative capacity is determined by

$$
\operatorname{CumC}^{\mathrm{x}}(f)=C^{\mathrm{x}}\left(L=\left\|\frac{f}{f_{s}}\right\|\right)
$$

where []$^{\mathrm{X}}$ denotes the examined scheme configuration -either SISO or SIMO or MISO or MIMO one- and $\|x\|$ means the nearest integer to $x$. 
In fact, cumulative capacity describes the aggregate capacity effect of all subchannels of the examined frequency band.

- The cumulative total average power consumption. Similarly to the cumulative capacity, cumulative total average power consumption can be defined as the cumulative upper limit of power consumption in Watts of UWA systems. For given frequency $f$, the cumulative total average power consumption is determined by

$$
C u m P_{t o t}^{\mathrm{X}}(f)=P_{t o t}\left(L=\left\|\frac{f}{f_{s}}\right\|\right)
$$

- The EE cumulative capacity. It defines an appropriate EE metric providing a macroscopic qualitative estimate of the role of IPSDM limits and system power consumption in UWA system operation. EE cumulative capacity denotes the cumulative upper limit of bits that the system can deliver per Joule consumed into the system. On the basis of eqs. (29) and (30), this EE capacity metric is given by:

$$
\operatorname{CumEEC}^{\mathrm{X}}(f)=\frac{\operatorname{CumC}^{\mathrm{X}}(f)}{\operatorname{CumP}_{\text {tot }}^{\mathrm{X}}(f)}
$$

\section{Discussion and Numerical Results}

The simulation results of various types of single- and multi-port UWA networks aim at investigating: (a) their broadband potential; (b) how SE and EE metrics are affected by the implementation of various MIMO schemes; (c) the SE/EE dynamic equilibria; (d) the influence of UWA modems through the different power consumption scenarios considered; and (e) the impact of UWA configuration parameters, IPSDM limits and noise conditions on the aforementioned SE/EE dynamic equilibria.

For the numerical computations, the UWA/MIMO configuration depicted in Fig. 1 has been considered. In order to apply the propagation and transmission analysis of Secs. II and III as well as the SE and EE performance metrics of Sec. V, UWA configuration parameters are reported in Table 1 . Note that the vast majority of these default parameters are derived from UWA/MIMO experiments (see also in [13], [56]).

As it has already been mentioned, the UWA channel is perfectly known to the receiver transducers since channel knowledge at them can be maintained via training and tracking. As it concerns the properties of metrics, the flat-fading subchannel frequency spacing $f_{s}$ and the number of subchannels $K$ in the UWA signal frequency range $0-100 \mathrm{kHz}$ are assumed equal to $100 \mathrm{~Hz}$ and 1000 , respectively.

As it concerns the power consumption of the involved UWA systems, the related circuit and system parameters, which are reported in Sec.IV and detailed in [18], [19], [48]-[50], are defined in Table II. These values correspond to the two different power consumption scenarios of Sec.IV and may provide a satisfactory approximation towards the actual UWA system power consumption.

\section{A. End-to-End Channel Attenuation and Spectral Capacity of UWA/SISO Channels}

The potential transmission, SE and EE performance of UWA/SISO channels in terms of attenuation, spectral capacity (i.e., either cumulative capacity or EE cumulative 
capacity) and power consumption in the $0-100 \mathrm{kHz}$ frequency range is assessed in this subsection.

In Fig. 3(a), the end-to-end channel attenuation of UWA/SISO channels (either $\mathrm{SISO} / \mathrm{CCs}$ or $\mathrm{SISO} / \mathrm{XCs}$ ) from transmit transducer $\mathrm{T}_{1}$ to receive transducers $\mathrm{R}_{j}, j=1, . ., 4$ is plotted with respect to frequency in the $0-100 \mathrm{kHz}$ frequency band. In Figs. 3(b)-(d), same plots are given in the case of the transmit transducer $\mathrm{T}_{2}, \mathrm{~T}_{3}$ and $\mathrm{T}_{4}$, respectively.

TABLE I

Default UWA/MIMO Configuration Parameters

\begin{tabular}{|c|c|c|c|c|c|}
\hline Letter & $\begin{array}{l}\text { Default } \\
\text { Value }\end{array}$ & Letter & $\begin{array}{l}\text { Default } \\
\text { Value }\end{array}$ & Letter & $\begin{array}{c}\text { Default } \\
\text { Value }\end{array}$ \\
\hline$D$ & $100 \mathrm{~m}$ & $n_{\mathrm{T}}$ & 4 & $n_{\mathrm{R}}$ & 4 \\
\hline$z_{\mathrm{T} 1}$ & $0 \mathrm{~m}$ & $y_{\mathrm{T} 1}$ & $9 \mathrm{~m}$ & $\Delta_{\mathrm{yT}}$ & $0.6 \mathrm{~m}$ \\
\hline$z_{\mathrm{R} 1}$ & $100 \mathrm{~m}$ & $y_{\mathrm{R} 1}$ & $9 \mathrm{~m}$ & $\Delta_{\mathrm{yR}}$ & $0.6 \mathrm{~m}$ \\
\hline$S p$ & 1.5 & $c$ & $1500 \mathrm{~m} / \mathrm{s}$ & $c_{1}$ & $1650 \mathrm{~m} / \mathrm{s}$ \\
\hline$\Delta_{\mathrm{zT}}=\Delta_{\mathrm{zR}}$ & $0 \mathrm{~m}$ & $p$ & $1023 \mathrm{~kg} / \mathrm{m}^{3}$ & $p_{1}$ & $1500 \mathrm{~kg} / \mathrm{m}^{3}$ \\
\hline$L_{S S}$ & $-0.5 \mathrm{~dB}$ & & & $L_{S B}$ & $-3 \mathrm{~dB}$ \\
\hline
\end{tabular}

TABLE II

Power Consumption UWA/MIMO System Parameters for the

Two Power Consumption Scenarios

\begin{tabular}{|c|c|c||c|c|c||c|c|c|}
\hline \multirow{2}{*}{ Letter } & \multicolumn{2}{|c|}{ Default Value } & Letter & \multicolumn{2}{c|}{ Default Value } & Letter & \multicolumn{2}{c|}{ Default Value } \\
\cline { 2 - 7 } & $\begin{array}{c}\text { Scenario } \\
\text { A }\end{array}$ & $\begin{array}{c}\text { Scenario } \\
\text { B }\end{array}$ & & $\begin{array}{c}\text { Scenario } \\
\text { A }\end{array}$ & $\begin{array}{c}\text { Scenario } \\
\text { B }\end{array}$ & & $\begin{array}{c}\text { Scenario } \\
\text { A }\end{array}$ & $\begin{array}{c}\text { Scenario } \\
\text { B }\end{array}$ \\
\hline$\Xi$ & 1.015 & 1.015 & $M_{1}$ & $40 \mathrm{~dB}$ & $40 \mathrm{~dB}$ & $P_{\text {filt }}$ & $2.5 \mathrm{~mW}$ & $2.5 \mathrm{~mW}$ \\
\hline$P_{L N A}$ & $20 \mathrm{~mW}$ & $20 \mathrm{~mW}$ & $n$ & 0.35 & 0.35 & $P_{D A C}$ & $15.4 \mathrm{~mW}$ & $15.4 \mathrm{~mW}$ \\
\hline$P_{\text {fil }}$ & $2.5 \mathrm{~mW}$ & $2.5 \mathrm{~mW}$ & $P_{I F A}$ & $3 \mathrm{~mW}$ & $3 \mathrm{~mW}$ & $N_{r}(f)=10+N(f)$ & $\mathrm{dBm} / \mathrm{Hz}$ & $\mathrm{dBm} / \mathrm{Hz}$ \\
\hline$P_{m i x}$ & $30.3 \mathrm{~mW}$ & - & $P_{\text {syn }}$ & $50 \mathrm{~mW}$ & - & $P_{A D C}$ & $6.7 \mathrm{~mW}$ & $6.7 \mathrm{~mW}$ \\
\hline
\end{tabular}




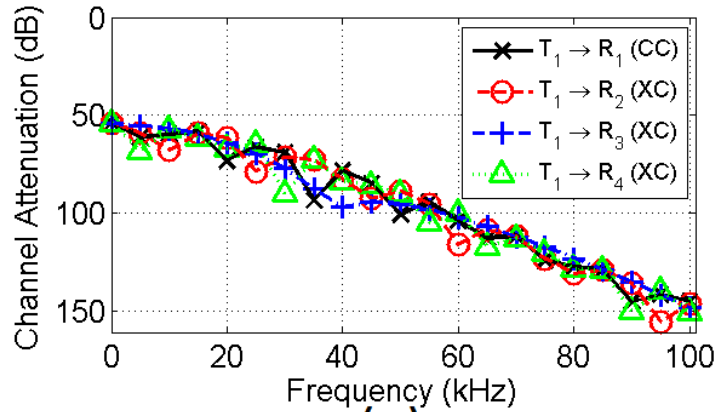

(a)

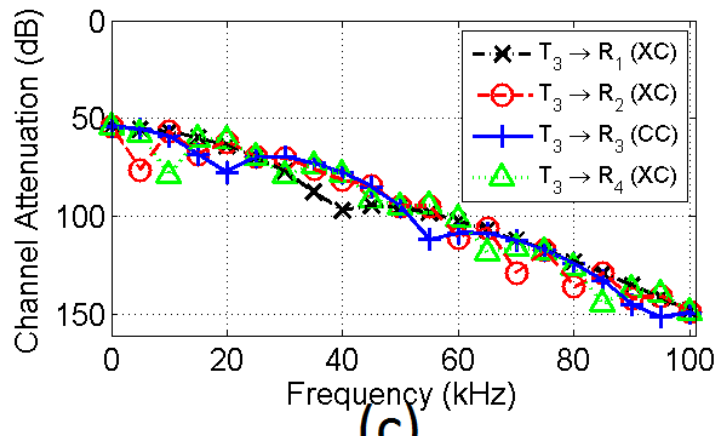

(c)

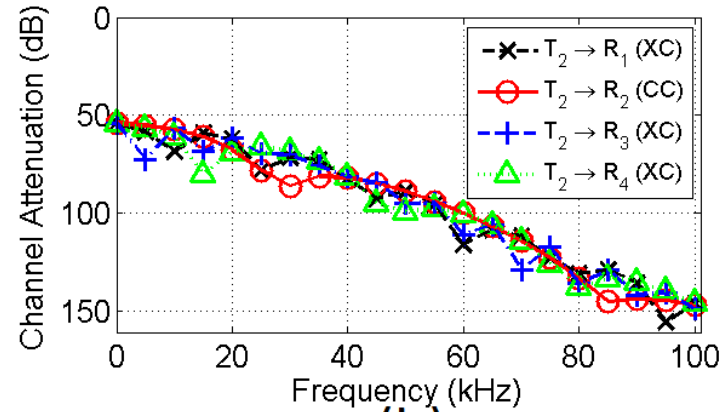

(b)

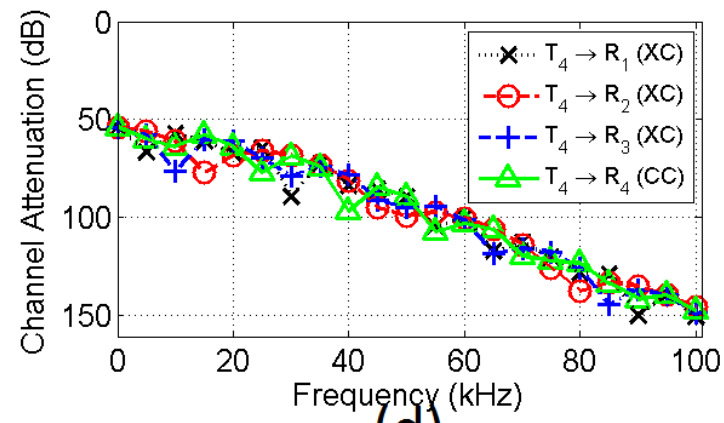

(d)

Fig. 3. Channel Attenuation of SISO/CCs (solid lines) and SISO/XCs (dashed lines) of the examined UWA configuration (for plot clarity reasons, the plot frequency spacing is equal to $5 \mathrm{kHz}$ ).

(a) Transmit transducer $T_{1}$. (b) Transmit transducer $T_{2}$.

(c) Transmit transducer $T_{3}$. (d) Transmit transducer $T_{4}$.

At the same time, to investigate the spectral behavior of the aforementioned UWA/SISO channels, the typical uniform IPSDM limits, presented in Sec.IVA, and the typical uniform AWGN PSD, presented in Sec.IVB, are considered when five indicative UWA topologies of different distances are examined (i.e., $z_{\mathrm{R} 1}=50 \mathrm{~m}, z_{\mathrm{R} 1}=100 \mathrm{~m}$, $z_{\mathrm{R} 1}=200 \mathrm{~m}, z_{\mathrm{R} 1}=500 \mathrm{~m}$ and $\left.z_{\mathrm{R} 1}=1000 \mathrm{~m}\right)$. Also, it is assumed that only the median values of spectral capacity over $\mathrm{CCs}$ and $\mathrm{XCs}$ for each of the examined indicative $\mathrm{UWA} / \mathrm{SISO}$ topologies is presented.

In Fig. 4(a), the median cumulative capacity of the SISO/CCs and SISO/XCs 


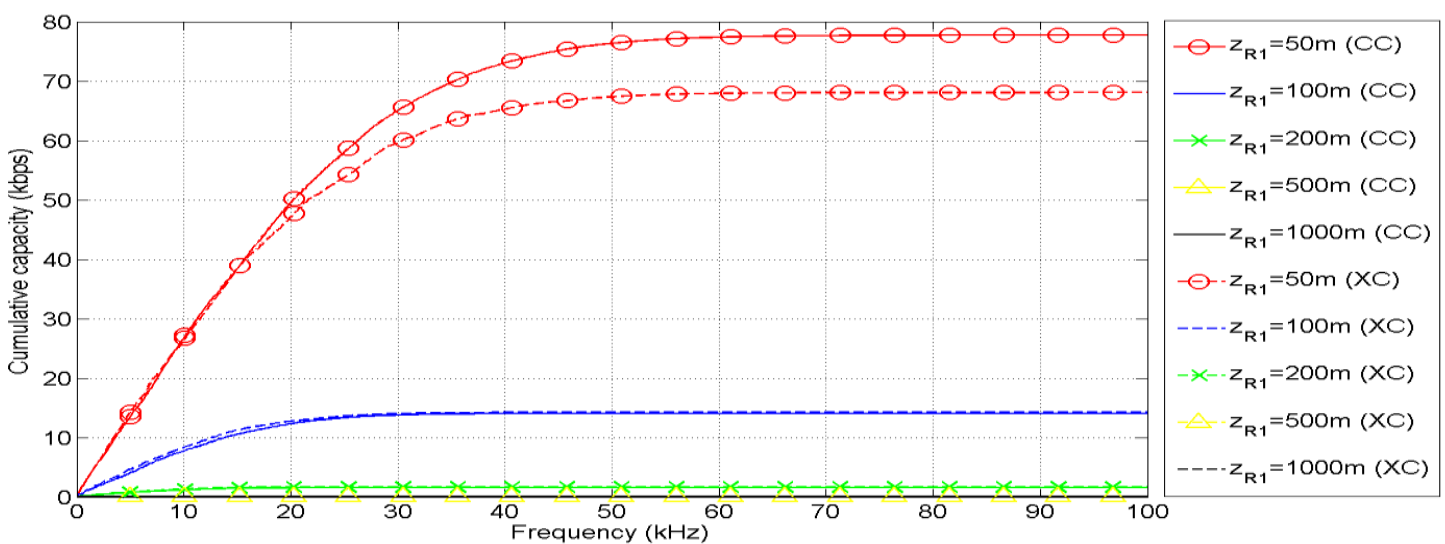

(a)
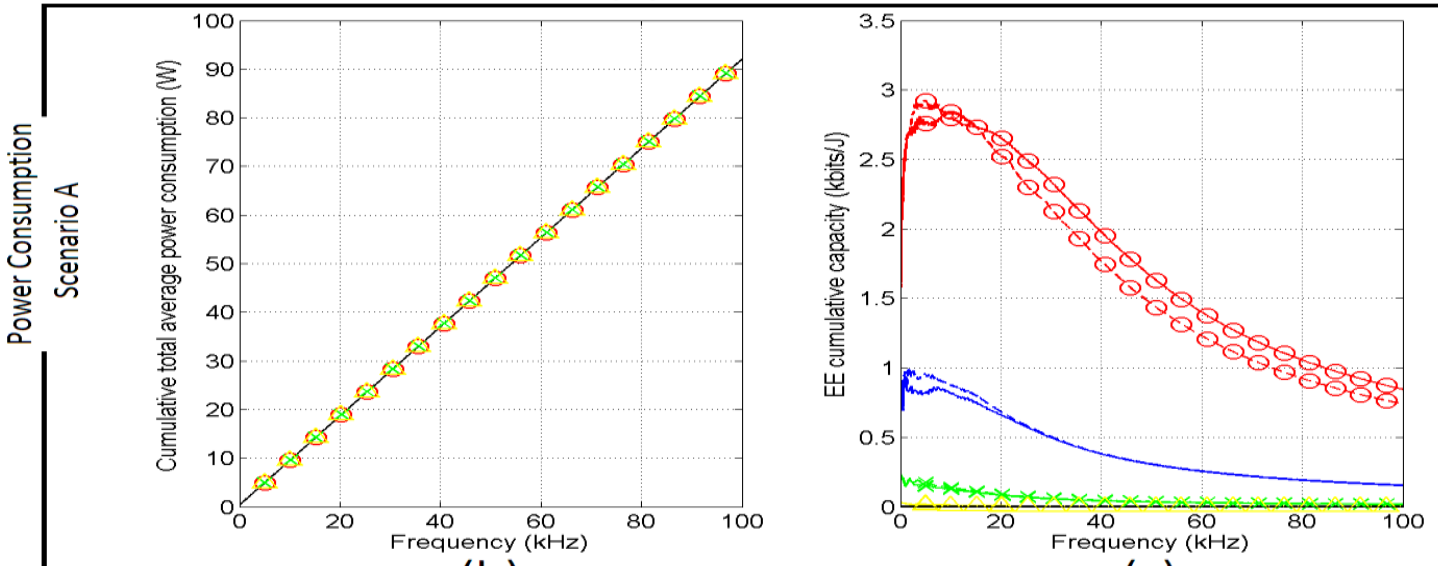

(b)

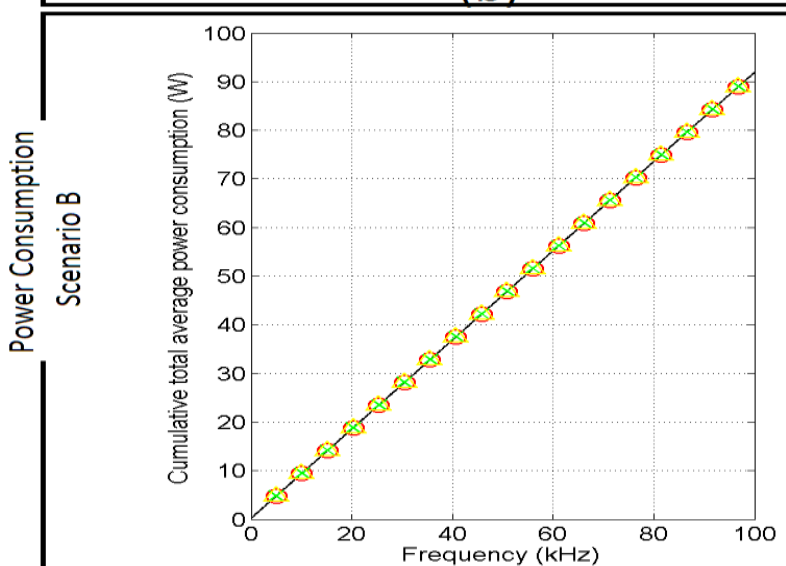

(d)

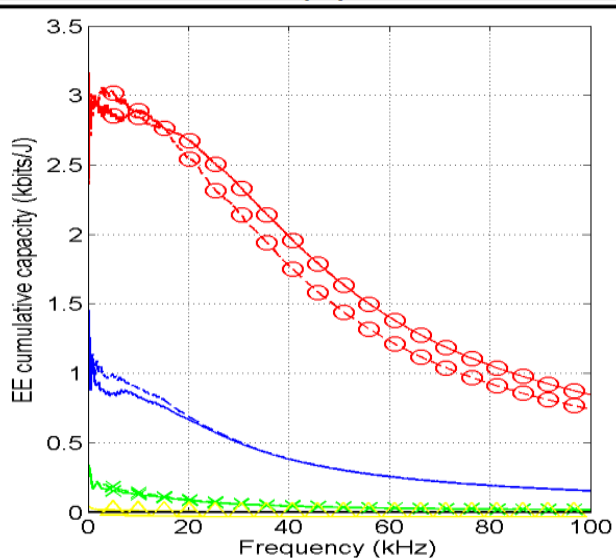

(e)

Fig. 4. Spectral capacity characteristics of SISO/CCs (solid lines) and SISO/XCs (dashed lines) of the examined UWA configuration for five indicative topologies (the subchannel frequency spacing is equal to $100 \mathrm{~Hz}$ ). (a) Median cumulative capacity. (b) Median cumulative total average power consumption. (c) Median EE cumulative capacity.

is plotted versus frequency in the $0-100 \mathrm{kHz}$ frequency range for both power consumption scenarios. As it concerns the EE performance of the examined power consumption scenarios, in Fig. 4(b), the median cumulative total average power consumption of these SISO channels is plotted with respect to frequency for the 
power consumption scenario A. In Fig. 4(c), the median EE cumulative capacity of these SISO channels is plotted with respect to frequency for the power consumption scenario A. In Figs. 4(d) and 4(e), respective curves with Figs. 4(b) and 4(c) are given but for the power consumption scenario B.

Observations that are based on Figs. 3(a)-(d) and 4(a)-(e) are made as follows [7], [10], [12], [13], [57].

- In contrast with other traditional wireless and wireline communications channels, UWA/SISO channels are characterized by a path loss that mainly depends on the distance between the transmit/receive transducers as well as the operating frequency. At the same time, the spreading losses of UWA channels severely increase with distance [7], [13]. Totally, the channel attenuation of UWA channels present significant frequency-dependency resembling the behavior of low-pass filters. Regarding the IoT and M2M applications, the behavior of UWA channels implies that high frequencies can only be dedicated for short-range narrowband UWA applications since distance and low-pass behavior pulverize their broadband potential.

- As it concerns the attenuation due to reflection losses, spectral notches are observed across the end-to-end channel attenuation. In contrast to traditional wireless and wireline communications channels where the later arriving rays carry less energy than the earlier ones, in UWA channels, it is often the case that the later arriving rays may carry more energy than the earlier ones [12]. Despite this multipath feature, UWA multipath environment versatility offers the appropriate basis for the deployment of MIMO networks so that the capacity potential of UWA channels can be further exploited.

- Despite the end-to-end channel attenuation similarities among SISO/CCs and $\mathrm{SISO} / \mathrm{XCs}$, there are differences that depend drastically on the frequency, the channel type -either $\mathrm{CC}$ or $\mathrm{XC}-$, the UWA configuration (i.e., horizontal and vertical spacings, water depth, etc), the physical properties of the transmission media and the end-to-end -"LOS"- distance. As it is going to be shown, this peculiar transmission behavior of UWA/SISO channels is reflected on their corresponding spectral metrics and the spectral behavior of multi-port UWA networks.

- As it concerns SE metrics of SISO channels in terms of cumulative capacity, the significantly high IPSDM limits combined with short average end-to-end transmission distances, low end-to-end channel attenuations and low noise environment characteristics can make their SE metrics attractive for short- and medium-range broadband UWA/IoT and UWA/M2M applications.

- In all the UWA topologies examined, SISO/CCs and SISO/XCs present the same cumulative total average power consumption due to the same number of involved transducers. As it concerns SE and EE metrics, CCs are those that statistically convey slightly higher metrics in comparison with XCs. This is due to the fact that the end-to-end distances as well as the additive ray path lengths present negligible differences among different UWA/SISO configurations. Anyway, this result also has to do with the UWA configuration geometry and the arrangement of the transmit and receive transducers.

- Despite the high IPSDM limits, the results of cumulative total average power consumption and EE cumulative capacity metrics reveal the inefficient use of 
power, especially above $5 \mathrm{kHz}$ regardless of the examined power consumption scenario. In order to mitigate this power waste, appropriate IPSDM limits and UWA/MIMO configurations are required to be designed so as to optimize: (i) the trade-off between SE and EE performance of the allocated UWA frequency spectrum; and (ii) EE intraoperability of different UWA configurations so that scalable capacities among different single- and multi-port UWA networks can be assured. Since power consumption scenario A and B describe the respective performance of today's and future's UWA transducers, the following optimization analysis becomes critical for the future development of UWA networks in undersea IoT and M2M communications.

\section{B. Single- and Multi-Port UWA Networks: SE and EE Performance}

Recently, growing concern arises from the need for increasing profitability through power consumption reduction and for controlling the environmental effect [18], [48], [58]. Until now, the key parameter during multi-port UWA network design was the selection of the optimum number of transmit and receive transducers, which succeeds the best trade-off between system complexity and capacity. However, the optimization problem is now differentiated by taking under consideration both $\mathrm{SE}$ and $\mathrm{EE}$ performance of multi-port UWA networks. In this subsection, the SE and EE performance of different multi-port UWA configurations is investigated when: (i) different IPSDM limits; (ii) different noise conditions; and (iii) different MIMO configuration properties; are applied.

Already identified in Sec.VIA, there is a great variety of possible arrangements that can be supported and examined for given multi-port scheme. In Table 3, all the possible arrangements are reported indicating that the analytical presentation of the $\mathrm{SE}$ and EE performance of each single- and multi-port arrangement is impracticable. To facilitate the analysis without harming its generality, in the rest of this paper, it is assumed that only the median values of each SISO/CC, SISO/XC, SIMO, MISO and MIMO scheme presented in Table 3 will be studied in the $0-100 \mathrm{kHz}$ frequency band. 
TABLE III

MIMO Schemes and Corresponding Arrangements

\begin{tabular}{|c|c|}
\hline Scheme & Arrangements \\
\hline $\mathrm{SISO} / \mathrm{CC}$ & $\mathrm{T}_{1} \rightarrow \mathrm{R}_{1} ; \mathrm{T}_{2} \rightarrow \mathrm{R}_{2} ; \mathrm{T}_{3} \rightarrow \mathrm{R}_{3} ; \mathrm{T}_{4} \rightarrow \mathrm{R}_{4}$ \\
\hline $\mathrm{SISO} / \mathrm{XC}$ & $\mathrm{T}_{1} \rightarrow \mathrm{R}_{2} ; \mathrm{T}_{1} \rightarrow \mathrm{R}_{3} ; \mathrm{T}_{1} \rightarrow \mathrm{R}_{4} ; \mathrm{T}_{2} \rightarrow \mathrm{R}_{1} ; \mathrm{T}_{2} \rightarrow \mathrm{R}_{3} ; \mathrm{T}_{2} \rightarrow \mathrm{R}_{4} ; \mathrm{T}_{3} \rightarrow \mathrm{R}_{1} ; \mathrm{T}_{3} \rightarrow \mathrm{R}_{2} ; \mathrm{T}_{3} \rightarrow \mathrm{R}_{4}$ \\
\hline 1x2 SIMO & $\begin{array}{c}\mathrm{T}_{1} \rightarrow \mathrm{R}_{1}, \mathrm{R}_{2} ; \mathrm{T}_{1} \rightarrow \mathrm{R}_{1}, \mathrm{R}_{3} ; \mathrm{T}_{1} \rightarrow \mathrm{R}_{1}, \mathrm{R}_{4} ; \mathrm{T}_{1} \rightarrow \mathrm{R}_{2}, \mathrm{R}_{3} ; \mathrm{T}_{1} \rightarrow \mathrm{R}_{2}, \mathrm{R}_{4} ; \mathrm{T}_{1} \rightarrow \mathrm{R}_{3}, \mathrm{R}_{4} ; \mathrm{T}_{2} \rightarrow \mathrm{R}_{1}, \mathrm{R}_{2} ; \mathrm{T}_{2} \rightarrow \mathrm{R}_{1}, \mathrm{R}_{3} ; \mathrm{T}_{2} \rightarrow \mathrm{R}_{1}, \mathrm{R}_{4} ; \\
\mathrm{T}_{2} \rightarrow \mathrm{R}_{2}, \mathrm{R}_{3} ; \mathrm{T}_{2} \rightarrow \mathrm{R}_{2}, \mathrm{R}_{4} ; \mathrm{T}_{2} \rightarrow \mathrm{R}_{3}, \mathrm{R}_{4} ; \mathrm{T}_{3} \rightarrow \mathrm{R}_{1}, \mathrm{R}_{2} ; \mathrm{T}_{3} \rightarrow \mathrm{R}_{1}, \mathrm{R}_{3} ; \mathrm{T}_{3} \rightarrow \mathrm{R}_{1}, \mathrm{R}_{4} ; \mathrm{T}_{3} \rightarrow \mathrm{R}_{2}, \mathrm{R}_{3} ; \mathrm{T}_{3} \rightarrow \mathrm{R}_{2}, \mathrm{R}_{4} ; \mathrm{T}_{3} \rightarrow \mathrm{R}_{3}, \mathrm{R}_{4} ; \\
\mathrm{T}_{4} \rightarrow \mathrm{R}_{1}, \mathrm{R}_{2} ; \mathrm{T}_{4} \rightarrow \mathrm{R}_{1}, \mathrm{R}_{3} ; \mathrm{T}_{4} \rightarrow \mathrm{R}_{1}, \mathrm{R}_{4} ; \mathrm{T}_{4} \rightarrow \mathrm{R}_{2}, \mathrm{R}_{3} ; \mathrm{T}_{4} \rightarrow \mathrm{R}_{2}, \mathrm{R}_{4} ; \mathrm{T}_{4} \rightarrow \mathrm{R}_{3}, \mathrm{R}_{4} ;\end{array}$ \\
\hline 1x3 SIMO & $\begin{array}{c}\mathrm{T}_{1} \rightarrow \mathrm{R}_{1}, \mathrm{R}_{2}, \mathrm{R}_{3} ; \mathrm{T}_{1} \rightarrow \mathrm{R}_{1}, \mathrm{R}_{2}, \mathrm{R}_{4} ; \mathrm{T}_{1} \rightarrow \mathrm{R}_{1}, \mathrm{R}_{3}, \mathrm{R}_{4} ; \mathrm{T}_{1} \rightarrow \mathrm{R}_{2}, \mathrm{R}_{3}, \mathrm{R}_{4} ; \mathrm{T}_{2} \rightarrow \mathrm{R}_{1}, \mathrm{R}_{2}, \mathrm{R}_{3} ; \mathrm{T}_{2} \rightarrow \mathrm{R}_{1}, \mathrm{R}_{2}, \mathrm{R}_{4} ; \mathrm{T}_{2} \rightarrow \mathrm{R}_{1}, \mathrm{R}_{3}, \mathrm{R}_{4} ; \\
\mathrm{T}_{2} \rightarrow \mathrm{R}_{2}, \mathrm{R}_{3}, \mathrm{R}_{4} ; \mathrm{T}_{3} \rightarrow \mathrm{R}_{1}, \mathrm{R}_{2}, \mathrm{R}_{3} ; \mathrm{T}_{3} \rightarrow \mathrm{R}_{1}, \mathrm{R}_{2}, \mathrm{R}_{4} ; \mathrm{T}_{3} \rightarrow \mathrm{R}_{1}, \mathrm{R}_{3}, \mathrm{R}_{4} ; \mathrm{T}_{3} \rightarrow \mathrm{R}_{2}, \mathrm{R}_{3}, \mathrm{R}_{4} ; \mathrm{T}_{4} \rightarrow \mathrm{R}_{1}, \mathrm{R}_{2}, \mathrm{R}_{3} ; \mathrm{T}_{4} \rightarrow \mathrm{R}_{1}, \mathrm{R}_{2}, \mathrm{R}_{4} ; \\
\mathrm{T}_{4} \rightarrow \mathrm{R}_{1}, \mathrm{R}_{3}, \mathrm{R}_{4} ; \mathrm{T}_{4} \rightarrow \mathrm{R}_{2}, \mathrm{R}_{3}, \mathrm{R}_{4} ;\end{array}$ \\
\hline $1 \times 4$ SIMO & $\mathrm{T}_{1} \rightarrow \mathrm{R}_{1}, \mathrm{R}_{2}, \mathrm{R}_{3}, \mathrm{R}_{4} ; \mathrm{T}_{2} \rightarrow \mathrm{R}_{1}, \mathrm{R}_{2}, \mathrm{R}_{3}, \mathrm{R}_{4} ; \mathrm{T}_{3} \rightarrow \mathrm{R}_{1}, \mathrm{R}_{2}, \mathrm{R}_{3}, \mathrm{R}_{4} ; \mathrm{T}_{4} \rightarrow \mathrm{R}_{1}, \mathrm{R}_{2}, \mathrm{R}_{3}, \mathrm{R}_{4}$ \\
\hline 2x1 MISO & $\begin{array}{c}\mathrm{T}_{1}, \mathrm{~T}_{2} \rightarrow \mathrm{R}_{1} ; \mathrm{T}_{1}, \mathrm{~T}_{3} \rightarrow \mathrm{R}_{1} ; \mathrm{T}_{1}, \mathrm{~T}_{4} \rightarrow \mathrm{R}_{1} ; \mathrm{T}_{2}, \mathrm{~T}_{3} \rightarrow \mathrm{R}_{1} ; \mathrm{T}_{2}, \mathrm{~T}_{4} \rightarrow \mathrm{R}_{1} ; \mathrm{T}_{3}, \mathrm{~T}_{4} \rightarrow \mathrm{R}_{1} ; \mathrm{T}_{1}, \mathrm{~T}_{2} \rightarrow \mathrm{R}_{2} ; \mathrm{T}_{1}, \mathrm{~T}_{3} \rightarrow \mathrm{R}_{2} ; \mathrm{T}_{1}, \mathrm{~T}_{4} \rightarrow \mathrm{R}_{2} ; \\
\mathrm{T}_{2}, \mathrm{~T}_{3} \rightarrow \mathrm{R}_{2} ; \mathrm{T}_{2}, \mathrm{~T}_{4} \rightarrow \mathrm{R}_{2} ; \mathrm{T}_{3}, \mathrm{~T}_{4} \rightarrow \mathrm{R}_{2} ; \mathrm{T}_{1}, \mathrm{~T}_{2} \rightarrow \mathrm{R}_{3} ; \mathrm{T}_{1}, \mathrm{~T}_{3} \rightarrow \mathrm{R}_{3} ; \mathrm{T}_{1}, \mathrm{~T}_{4} \rightarrow \mathrm{R}_{3} ; \mathrm{T}_{2}, \mathrm{~T}_{3} \rightarrow \mathrm{R}_{3} ; \mathrm{T}_{2}, \mathrm{~T}_{4} \rightarrow \mathrm{R}_{3} ; \mathrm{T}_{3}, \mathrm{~T}_{4} \rightarrow \mathrm{R}_{3} ; \\
\mathrm{T}_{1}, \mathrm{~T}_{2} \rightarrow \mathrm{R}_{4} ; \mathrm{T}_{1}, \mathrm{~T}_{3} \rightarrow \mathrm{R}_{4} ; \mathrm{T}_{1}, \mathrm{~T}_{4} \rightarrow \mathrm{R}_{4} ; \mathrm{T}_{2}, \mathrm{~T}_{3} \rightarrow \mathrm{R}_{4} ; \mathrm{T}_{2}, \mathrm{~T}_{4} \rightarrow \mathrm{R}_{4} ; \mathrm{T}_{3}, \mathrm{~T}_{4} \rightarrow \mathrm{R}_{4} ;\end{array}$ \\
\hline $3 \times 1 \mathrm{MISO}$ & $\begin{array}{c}\mathrm{T}_{1}, \mathrm{~T}_{2}, \mathrm{~T}_{3} \rightarrow \mathrm{R}_{1} ; \mathrm{T}_{1}, \mathrm{~T}_{2}, \mathrm{~T}_{4} \rightarrow \mathrm{R}_{1} ; \mathrm{T}_{1}, \mathrm{~T}_{3}, \mathrm{~T}_{4} \rightarrow \mathrm{R}_{1} ; \mathrm{T}_{2}, \mathrm{~T}_{3}, \mathrm{~T}_{4} \rightarrow \mathrm{R}_{1} ; \mathrm{T}_{1}, \mathrm{~T}_{2}, \mathrm{~T}_{3} \rightarrow \mathrm{R}_{2} ; \mathrm{T}_{1}, \mathrm{~T}_{2}, \mathrm{~T}_{4} \rightarrow \mathrm{R}_{2} ; \mathrm{T}_{1}, \mathrm{~T}_{2}, \mathrm{~T}_{4} \rightarrow \mathrm{R}_{3} ; \\
\mathrm{T}_{2}, \mathrm{~T}_{3}, \mathrm{~T}_{4} \rightarrow \mathrm{R}_{2} ; \mathrm{T}_{1}, \mathrm{~T}_{2}, \mathrm{~T}_{3} \rightarrow \mathrm{R}_{3} ; \mathrm{T}_{1}, \mathrm{~T}_{2}, \mathrm{~T}_{4} \rightarrow \mathrm{R}_{3} ; \mathrm{T}_{1}, \mathrm{~T}_{3}, \mathrm{~T}_{4} \rightarrow \mathrm{R}_{3} ; \mathrm{T}_{2}, \mathrm{~T}_{3}, \mathrm{~T}_{4} \rightarrow \mathrm{R}_{3} ; \mathrm{T}_{1}, \mathrm{~T}_{2}, \mathrm{~T}_{3} \rightarrow \mathrm{R}_{4} ; \mathrm{T}_{1}, \mathrm{~T}_{2}, \mathrm{~T}_{4} \rightarrow \mathrm{R}_{4} ; \\
\mathrm{T}_{1}, \mathrm{~T}_{2}, \mathrm{~T}_{4} \rightarrow \mathrm{R}_{4} ; \mathrm{T}_{2}, \mathrm{~T}_{3}, \mathrm{~T}_{4} \rightarrow \mathrm{R}_{4} ;\end{array}$ \\
\hline 4x1 MISO & $\mathrm{T}_{1}, \mathrm{~T}_{2}, \mathrm{~T}_{3}, \mathrm{~T}_{4} \rightarrow \mathrm{R}_{1} ; \mathrm{T}_{1}, \mathrm{~T}_{2}, \mathrm{~T}_{3}, \mathrm{~T}_{4} \rightarrow \mathrm{R}_{2} ; \mathrm{T}_{1}, \mathrm{~T}_{2}, \mathrm{~T}_{3}, \mathrm{~T}_{4} \rightarrow \mathrm{R}_{3} ; \mathrm{T}_{1}, \mathrm{~T}_{2}, \mathrm{~T}_{3}, \mathrm{~T}_{4} \rightarrow \mathrm{R}_{4}$ \\
\hline $2 \times 2$ MIMO & $\begin{array}{l}\mathrm{T}_{1}, \mathrm{~T}_{2} \rightarrow \mathrm{R}_{1}, \mathrm{R}_{2} ; \mathrm{T}_{1}, \mathrm{~T}_{2} \rightarrow \mathrm{R}_{1}, \mathrm{R}_{3} ; \mathrm{T}_{1}, \mathrm{~T}_{2} \rightarrow \mathrm{R}_{1}, \mathrm{R}_{4} ; \mathrm{T}_{1}, \mathrm{~T}_{2} \rightarrow \mathrm{R}_{2}, \mathrm{R}_{3} ; \mathrm{T}_{1}, \mathrm{~T}_{2} \rightarrow \mathrm{R}_{2}, \mathrm{R}_{4} ; \mathrm{T}_{1}, \mathrm{~T}_{2} \rightarrow \mathrm{R}_{3}, \mathrm{R}_{4} \\
\mathrm{~T}_{1}, \mathrm{~T}_{3} \rightarrow \mathrm{R}_{1}, \mathrm{R}_{2} ; \mathrm{T}_{1}, \mathrm{~T}_{3} \rightarrow \mathrm{R}_{1}, \mathrm{R}_{3} ; \mathrm{T}_{1}, \mathrm{~T}_{3} \rightarrow \mathrm{R}_{1}, \mathrm{R}_{4} ; \mathrm{T}_{1}, \mathrm{~T}_{3} \rightarrow \mathrm{R}_{2}, \mathrm{R}_{3} ; \mathrm{T}_{1}, \mathrm{~T}_{3} \rightarrow \mathrm{R}_{2}, \mathrm{R}_{4} ; \mathrm{T}_{1}, \mathrm{~T}_{3} \rightarrow \mathrm{R}_{3}, \mathrm{R}_{4} ; \\
\mathrm{T}_{1}, \mathrm{~T}_{4} \rightarrow \mathrm{R}_{1}, \mathrm{R}_{2} ; \mathrm{T}_{1}, \mathrm{~T}_{4} \rightarrow \mathrm{R}_{1}, \mathrm{R}_{3} ; \mathrm{T}_{1}, \mathrm{~T}_{4} \rightarrow \mathrm{R}_{1}, \mathrm{R}_{4} ; \mathrm{T}_{1}, \mathrm{~T}_{4} \rightarrow \mathrm{R}_{2}, \mathrm{R}_{3} ; \mathrm{T}_{1}, \mathrm{~T}_{4} \rightarrow \mathrm{R}_{2}, \mathrm{R}_{4} ; \mathrm{T}_{1}, \mathrm{~T}_{4} \rightarrow \mathrm{R}_{3}, \mathrm{R}_{4} \\
\mathrm{~T}_{2}, \mathrm{~T}_{3} \rightarrow \mathrm{R}_{1}, \mathrm{R}_{2} ; \mathrm{T}_{2}, \mathrm{~T}_{3} \rightarrow \mathrm{R}_{1}, \mathrm{R}_{3} ; \mathrm{T}_{2}, \mathrm{~T}_{3} \rightarrow \mathrm{R}_{1}, \mathrm{R}_{4} ; \mathrm{T}_{2}, \mathrm{~T}_{3} \rightarrow \mathrm{R}_{2}, \mathrm{R}_{3} ; \mathrm{T}_{2}, \mathrm{~T}_{3} \rightarrow \mathrm{R}_{2}, \mathrm{R}_{4} ; \mathrm{T}_{2}, \mathrm{~T}_{3} \rightarrow \mathrm{R}_{3}, \mathrm{R}_{4} ; \\
\mathrm{T}_{2}, \mathrm{~T}_{4} \rightarrow \mathrm{R}_{1}, \mathrm{R}_{2} ; \mathrm{T}_{2}, \mathrm{~T}_{4} \rightarrow \mathrm{R}_{1}, \mathrm{R}_{3} ; \mathrm{T}_{2}, \mathrm{~T}_{4} \rightarrow \mathrm{R}_{1}, \mathrm{R}_{4} ; \mathrm{T}_{2}, \mathrm{~T}_{4} \rightarrow \mathrm{R}_{2}, \mathrm{R}_{3} ; \mathrm{T}_{2}, \mathrm{~T}_{4} \rightarrow \mathrm{R}_{2}, \mathrm{R}_{4} ; \mathrm{T}_{2}, \mathrm{~T}_{4} \rightarrow \mathrm{R}_{3}, \mathrm{R}_{4} \\
\mathrm{~T}_{3}, \mathrm{~T}_{4} \rightarrow \mathrm{R}_{1}, \mathrm{R}_{2} ; \mathrm{T}_{3}, \mathrm{~T}_{4} \rightarrow \mathrm{R}_{1}, \mathrm{R}_{3} ; \mathrm{T}_{3}, \mathrm{~T}_{4} \rightarrow \mathrm{R}_{1}, \mathrm{R}_{4} ; \mathrm{T}_{3}, \mathrm{~T}_{4} \rightarrow \mathrm{R}_{2}, \mathrm{R}_{3} ; \mathrm{T}_{3}, \mathrm{~T}_{4} \rightarrow \mathrm{R}_{2}, \mathrm{R}_{4} ; \mathrm{T}_{3}, \mathrm{~T}_{4} \rightarrow \mathrm{R}_{3}, \mathrm{R}_{4}\end{array}$ \\
\hline $2 \times 3 \mathrm{MIMO}$ & $\begin{array}{r}\mathrm{T}_{1}, \mathrm{~T}_{2} \rightarrow \mathrm{R}_{1}, \mathrm{R}_{2}, \mathrm{R}_{3} ; \mathrm{T}_{1}, \mathrm{~T}_{2} \rightarrow \mathrm{R}_{1}, \mathrm{R}_{2}, \mathrm{R}_{4} ; \mathrm{T}_{1}, \mathrm{~T}_{2} \rightarrow \mathrm{R}_{1}, \mathrm{R}_{3}, \mathrm{R}_{4} ; \mathrm{T}_{1}, \mathrm{~T}_{2} \rightarrow \mathrm{R}_{2}, \mathrm{R}_{3}, \mathrm{R}_{4} ; \mathrm{T}_{1}, \mathrm{~T}_{3} \rightarrow \mathrm{R}_{1}, \mathrm{R}_{2}, \mathrm{R}_{3} \\
\mathrm{~T}_{1}, \mathrm{~T}_{3} \rightarrow \mathrm{R}_{1}, \mathrm{R}_{2}, \mathrm{R}_{4} ; \mathrm{T}_{1}, \mathrm{~T}_{3} \rightarrow \mathrm{R}_{1}, \mathrm{R}_{3}, \mathrm{R}_{4} ; \mathrm{T}_{1}, \mathrm{~T}_{3} \rightarrow \mathrm{R}_{2}, \mathrm{R}_{3}, \mathrm{R}_{4} ; \mathrm{T}_{1}, \mathrm{~T}_{4} \rightarrow \mathrm{R}_{1}, \mathrm{R}_{2}, \mathrm{R}_{3} ; \mathrm{T}_{1}, \mathrm{~T}_{4} \rightarrow \mathrm{R}_{1}, \mathrm{R}_{2}, \mathrm{R}_{4} ; \\
\mathrm{T}_{1}, \mathrm{~T}_{4} \rightarrow \mathrm{R}_{1}, \mathrm{R}_{3}, \mathrm{R}_{4} ; \mathrm{T}_{1}, \mathrm{~T}_{4} \rightarrow \mathrm{R}_{2}, \mathrm{R}_{3}, \mathrm{R}_{4} ; \mathrm{T}_{2}, \mathrm{~T}_{3} \rightarrow \mathrm{R}_{1}, \mathrm{R}_{2}, \mathrm{R}_{3} ; \mathrm{T}_{2}, \mathrm{~T}_{3} \rightarrow \mathrm{R}_{1}, \mathrm{R}_{2}, \mathrm{R}_{4} ; \mathrm{T}_{2}, \mathrm{~T}_{3} \rightarrow \mathrm{R}_{1}, \mathrm{R}_{3}, \mathrm{R}_{4} ; \\
\mathrm{T}_{2}, \mathrm{~T}_{3} \rightarrow \mathrm{R}_{2}, \mathrm{R}_{3}, \mathrm{R}_{4} ; \mathrm{T}_{2}, \mathrm{~T}_{4} \rightarrow \mathrm{R}_{1}, \mathrm{R}_{2}, \mathrm{R}_{3} ; \mathrm{T}_{2}, \mathrm{~T}_{4} \rightarrow \mathrm{R}_{1}, \mathrm{R}_{2}, \mathrm{R}_{4} ; \mathrm{T}_{2}, \mathrm{~T}_{4} \rightarrow \mathrm{R}_{1}, \mathrm{R}_{3}, \mathrm{R}_{4} ; \mathrm{T}_{2}, \mathrm{~T}_{4} \rightarrow \mathrm{R}_{2}, \mathrm{R}_{3}, \mathrm{R}_{4} ; \\
\mathrm{T}_{3}, \mathrm{~T}_{4} \rightarrow \mathrm{R}_{1}, \mathrm{R}_{2}, \mathrm{R}_{3} ; \mathrm{T}_{3}, \mathrm{~T}_{4} \rightarrow \mathrm{R}_{1}, \mathrm{R}_{2}, \mathrm{R}_{4} ; \mathrm{T}_{3}, \mathrm{~T}_{4} \rightarrow \mathrm{R}_{1}, \mathrm{R}_{3}, \mathrm{R}_{4} ; \mathrm{T}_{3}, \mathrm{~T}_{4} \rightarrow \mathrm{R}_{2}, \mathrm{R}_{3}, \mathrm{R}_{4} ; \\
\end{array}$ \\
\hline 2x4 MIMO & $\begin{array}{c}\mathrm{T}_{1}, \mathrm{~T}_{2} \rightarrow \mathrm{R}_{1}, \mathrm{R}_{2}, \mathrm{R}_{3}, \mathrm{R}_{4} ; \mathrm{T}_{1}, \mathrm{~T}_{3} \rightarrow \mathrm{R}_{1}, \mathrm{R}_{2}, \mathrm{R}_{3}, \mathrm{R}_{4} ; \mathrm{T}_{1}, \mathrm{~T}_{4} \rightarrow \mathrm{R}_{1}, \mathrm{R}_{2}, \mathrm{R}_{3}, \mathrm{R}_{4} ; \mathrm{T}_{2}, \mathrm{~T}_{3} \rightarrow \mathrm{R}_{1}, \mathrm{R}_{2}, \mathrm{R}_{3}, \mathrm{R}_{4} ; \mathrm{T}_{2}, \mathrm{~T}_{4} \rightarrow \mathrm{R}_{1}, \mathrm{R}_{2}, \mathrm{R}_{3}, \mathrm{R}_{4} ; \\
\mathrm{T}_{3}, \mathrm{~T}_{4} \rightarrow \mathrm{R}_{1}, \mathrm{R}_{2}, \mathrm{R}_{3}, \mathrm{R}_{4} ;\end{array}$ \\
\hline $3 \times 2$ MIMO & $\begin{array}{l}\mathrm{T}_{1}, \mathrm{~T}_{2}, \mathrm{~T}_{3} \rightarrow \mathrm{R}_{1}, \mathrm{R}_{2} ; \mathrm{T}_{1}, \mathrm{~T}_{2}, \mathrm{~T}_{3} \rightarrow \mathrm{R}_{1}, \mathrm{R}_{3} ; \mathrm{T}_{1}, \mathrm{~T}_{2}, \mathrm{~T}_{3} \rightarrow \mathrm{R}_{1}, \mathrm{R}_{4} ; \mathrm{T}_{1}, \mathrm{~T}_{2}, \mathrm{~T}_{3} \rightarrow \mathrm{R}_{2}, \mathrm{R}_{3} ; \mathrm{T}_{1}, \mathrm{~T}_{2}, \mathrm{~T}_{3} \rightarrow \mathrm{R}_{2}, \mathrm{R}_{4} ; \mathrm{T}_{1}, \mathrm{~T}_{2}, \mathrm{~T}_{3} \rightarrow \mathrm{R}_{3}, \mathrm{R}_{4} ; \\
\mathrm{T}_{1}, \mathrm{~T}_{2}, \mathrm{~T}_{4} \rightarrow \mathrm{R}_{1}, \mathrm{R}_{2} ; \mathrm{T}_{1}, \mathrm{~T}_{2}, \mathrm{~T}_{4} \rightarrow \mathrm{R}_{1}, \mathrm{R}_{3} ; \mathrm{T}_{1}, \mathrm{~T}_{2}, \mathrm{~T}_{4} \rightarrow \mathrm{R}_{1}, \mathrm{R}_{4} ; \mathrm{T}_{1}, \mathrm{~T}_{2}, \mathrm{~T}_{4} \rightarrow \mathrm{R}_{2}, \mathrm{R}_{3} ; \mathrm{T}_{1}, \mathrm{~T}_{2}, \mathrm{~T}_{4} \rightarrow \mathrm{R}_{2}, \mathrm{R}_{4} ; \mathrm{T}_{1}, \mathrm{~T}_{2}, \mathrm{~T}_{4} \rightarrow \mathrm{R}_{3}, \mathrm{R}_{4} ; \\
\mathrm{T}_{1}, \mathrm{~T}_{3}, \mathrm{~T}_{4} \rightarrow \mathrm{R}_{1}, \mathrm{R}_{2} ; \mathrm{T}_{1}, \mathrm{~T}_{3}, \mathrm{~T}_{4} \rightarrow \mathrm{R}_{1}, \mathrm{R}_{3} ; \mathrm{T}_{1}, \mathrm{~T}_{3}, \mathrm{~T}_{4} \rightarrow \mathrm{R}_{1}, \mathrm{R}_{4} ; \mathrm{T}_{1}, \mathrm{~T}_{3}, \mathrm{~T}_{4} \rightarrow \mathrm{R}_{2}, \mathrm{R}_{3} ; \mathrm{T}_{1}, \mathrm{~T}_{3}, \mathrm{~T}_{4} \rightarrow \mathrm{R}_{2}, \mathrm{R}_{4} ; \mathrm{T}_{1}, \mathrm{~T}_{3}, \mathrm{~T}_{4} \rightarrow \mathrm{R}_{3}, \mathrm{R}_{4} ; \\
\mathrm{T}_{2}, \mathrm{~T}_{3}, \mathrm{~T}_{4} \rightarrow \mathrm{R}_{1}, \mathrm{R}_{2} ; \mathrm{T}_{2}, \mathrm{~T}_{3}, \mathrm{~T}_{4} \rightarrow \mathrm{R}_{1}, \mathrm{R}_{3} ; \mathrm{T}_{2}, \mathrm{~T}_{3}, \mathrm{~T}_{4} \rightarrow \mathrm{R}_{1}, \mathrm{R}_{4} ; \mathrm{T}_{2}, \mathrm{~T}_{3}, \mathrm{~T}_{4} \rightarrow \mathrm{R}_{2}, \mathrm{R}_{3} ; \mathrm{T}_{2}, \mathrm{~T}_{3}, \mathrm{~T}_{4} \rightarrow \mathrm{R}_{2}, \mathrm{R}_{4} ; \mathrm{T}_{2}, \mathrm{~T}_{3}, \mathrm{~T}_{4} \rightarrow \mathrm{R}_{3}, \mathrm{R}_{4} ;\end{array}$ \\
\hline $3 \times 3$ MIMO & $\begin{array}{l}\mathrm{T}_{1}, \mathrm{~T}_{2}, \mathrm{~T}_{3} \rightarrow \mathrm{R}_{1}, \mathrm{R}_{2}, \mathrm{R}_{3} ; \mathrm{T}_{1}, \mathrm{~T}_{2}, \mathrm{~T}_{3} \rightarrow \mathrm{R}_{1}, \mathrm{R}_{2}, \mathrm{R}_{4} ; \mathrm{T}_{1}, \mathrm{~T}_{2}, \mathrm{~T}_{3} \rightarrow \mathrm{R}_{1}, \mathrm{R}_{3}, \mathrm{R}_{4} ; \mathrm{T}_{1}, \mathrm{~T}_{2}, \mathrm{~T}_{3} \rightarrow \mathrm{R}_{2}, \mathrm{R}_{3}, \mathrm{R}_{4} \\
\mathrm{~T}_{1}, \mathrm{~T}_{2}, \mathrm{~T}_{4} \rightarrow \mathrm{R}_{1}, \mathrm{R}_{2}, \mathrm{R}_{3} ; \mathrm{T}_{1}, \mathrm{~T}_{2}, \mathrm{~T}_{4} \rightarrow \mathrm{R}_{1}, \mathrm{R}_{2}, \mathrm{R}_{4} ; \mathrm{T}_{1}, \mathrm{~T}_{2}, \mathrm{~T}_{4} \rightarrow \mathrm{R}_{1}, \mathrm{R}_{3}, \mathrm{R}_{4} ; \mathrm{T}_{1}, \mathrm{~T}_{2}, \mathrm{~T}_{4} \rightarrow \mathrm{R}_{2}, \mathrm{R}_{3}, \mathrm{R}_{4} \\
\mathrm{~T}_{1}, \mathrm{~T}_{3}, \mathrm{~T}_{4} \rightarrow \mathrm{R}_{1}, \mathrm{R}_{2}, \mathrm{R}_{3} ; \mathrm{T}_{1}, \mathrm{~T}_{3}, \mathrm{~T}_{4} \rightarrow \mathrm{R}_{1}, \mathrm{R}_{2}, \mathrm{R}_{4} ; \mathrm{T}_{1}, \mathrm{~T}_{3}, \mathrm{~T}_{4} \rightarrow \mathrm{R}_{1}, \mathrm{R}_{3}, \mathrm{R}_{4} ; \mathrm{T}_{1}, \mathrm{~T}_{3}, \mathrm{~T}_{4} \rightarrow \mathrm{R}_{2}, \mathrm{R}_{3}, \mathrm{R}_{4} \\
\mathrm{~T}_{2}, \mathrm{~T}_{3}, \mathrm{~T}_{4} \rightarrow \mathrm{R}_{1}, \mathrm{R}_{2}, \mathrm{R}_{3} ; \mathrm{T}_{2}, \mathrm{~T}_{3}, \mathrm{~T}_{4} \rightarrow \mathrm{R}_{1}, \mathrm{R}_{2}, \mathrm{R}_{4} ; \mathrm{T}_{2}, \mathrm{~T}_{3}, \mathrm{~T}_{4} \rightarrow \mathrm{R}_{1}, \mathrm{R}_{3}, \mathrm{R}_{4} ; \mathrm{T}_{2}, \mathrm{~T}_{3}, \mathrm{~T}_{4} \rightarrow \mathrm{R}_{2}, \mathrm{R}_{3}, \mathrm{R}_{4}\end{array}$ \\
\hline 4x3 MIMO & $\mathrm{T}_{1}, \mathrm{~T}_{2}, \mathrm{~T}_{3}, \mathrm{~T}_{4} \rightarrow \mathrm{R}_{1}, \mathrm{R}_{2}, \mathrm{R}_{3} ; \mathrm{T}_{1}, \mathrm{~T}_{2}, \mathrm{~T}_{3}, \mathrm{~T}_{4} \rightarrow \mathrm{R}_{1}, \mathrm{R}_{2}, \mathrm{R}_{4} ; \mathrm{T}_{1}, \mathrm{~T}_{2}, \mathrm{~T}_{3}, \mathrm{~T}_{4} \rightarrow \mathrm{R}_{1}, \mathrm{R}_{3}, \mathrm{R}_{4} ; \mathrm{T}_{1}, \mathrm{~T}_{2}, \mathrm{~T}_{3}, \mathrm{~T}_{4} \rightarrow \mathrm{R}_{2}, \mathrm{R}_{3}, \mathrm{R}_{4}$ \\
\hline 4x4 MIMO & $\mathrm{T}_{1}, \mathrm{~T}_{2}, \mathrm{~T}_{3}, \mathrm{~T}_{4} \rightarrow \mathrm{R}_{1}, \mathrm{R}_{2}, \mathrm{R}_{3}, \mathrm{R}_{4}$ \\
\hline
\end{tabular}

Combining either Fig. 4(a) with 4(c) or Fig. 4(a) with 4(e), an interesting $\mathrm{SE} / \mathrm{EE}$ trade-off relation can be proposed; in Fig. 5(a), the median EE capacity of $\mathrm{SISO} / \mathrm{CC}$ and $\mathrm{SISO} / \mathrm{XC}$ schemes is plotted versus the respective median capacities when IPSDM limits range from $-100 \mathrm{dBm} / \mathrm{Hz}$ to $50 \mathrm{dBm} / \mathrm{Hz}$ with step $1 \mathrm{dBm} / \mathrm{Hz}$ for the power consumption scenario A. The default IPSDM limits are also denoted here. In Figs. 5(b) and 5(c), same curves are given with Fig. 5(a) but for the SIMO (i.e., 1x2, 1x3 and 1x4) 


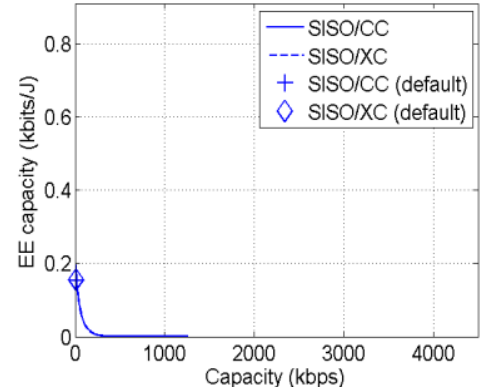

(a)

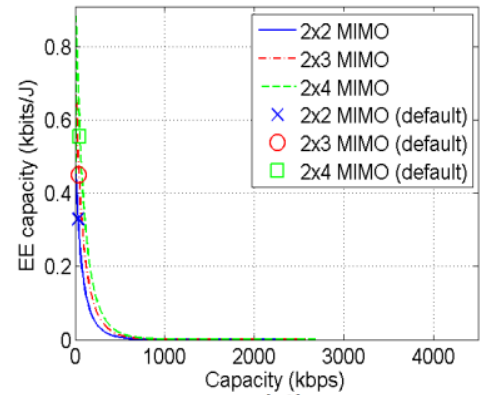

(d)

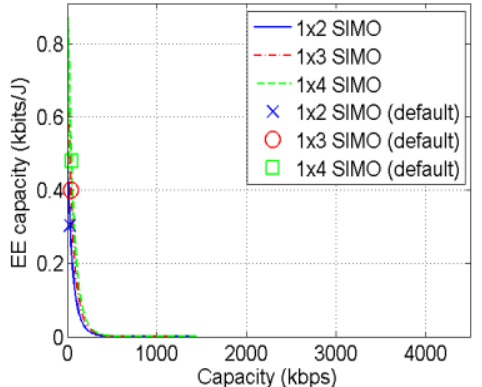

(b)

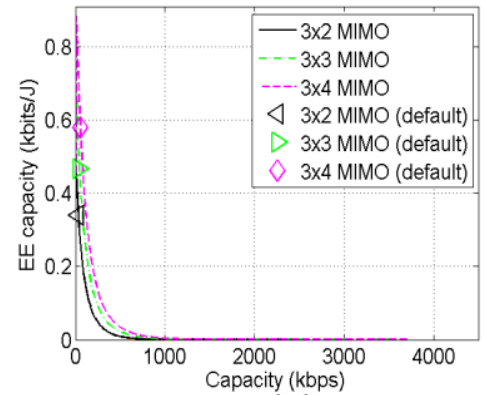

(e)

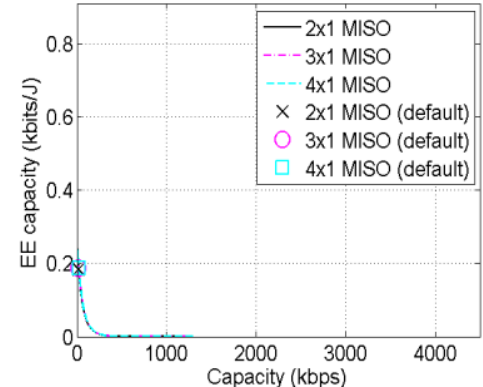

(c)

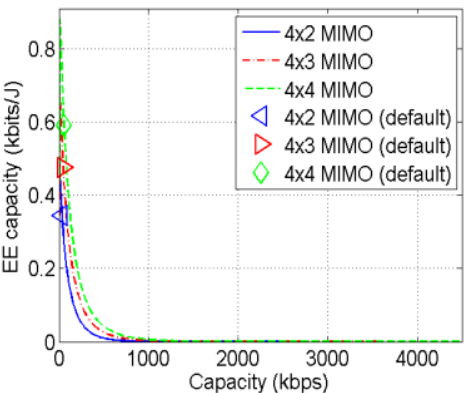

(f)

Fig. 5. Trade-off curves between median EE capacity and median capacity for various singleand multi-port schemes when different IPSDM limits are adopted for the power consumption scenario A.

(a) SISO/CC and SISO/XC trade-off curves. (b) SIMO trade-off curves.

(c) MISO trade-off curves. (d)-(f) MIMO trade-off curves.

and MISO (i.e., 2x1, 3x1 and 4x1) schemes, respectively. Similarly to Figs. 5(a)-(c) and based on the proposed SE/EE trade-off relation, a plethora of MIMO schemes (i.e., 2x2, $2 \times 3,2 \times 4,3 \times 2,3 \times 3,3 \times 4$ and $4 \times 4)$ is studied in Figs. 5(d)-(f). In Figs. 6(a)-(f), same curves are plotted with Figs. 5(a)-(f) but for the power consumption scenario B.

Comparing Figs. 5(a)-(f) and 6(a)-(f) with the previous figures, several interesting remarks can be pointed out:

- The today's state-of-the-art research topic in UWA technology is the adoption of MIMO principles across the deployed UWA networks [10], [11], [13]. MIMO transmission methods may be applied to UWA networks permitting a boost of their SE and EE capacity by appropriately exploiting their transmit and receive transducers. 


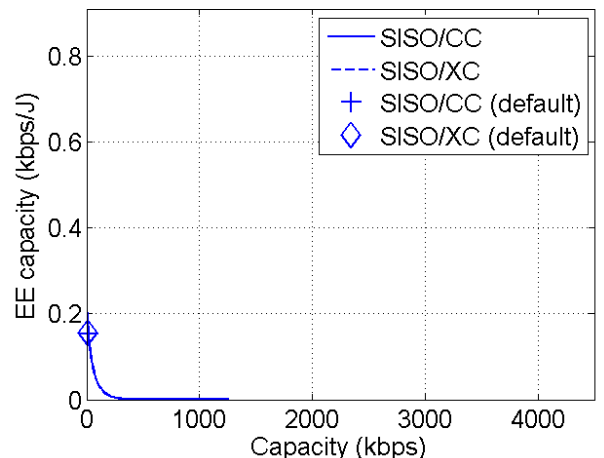

(a)

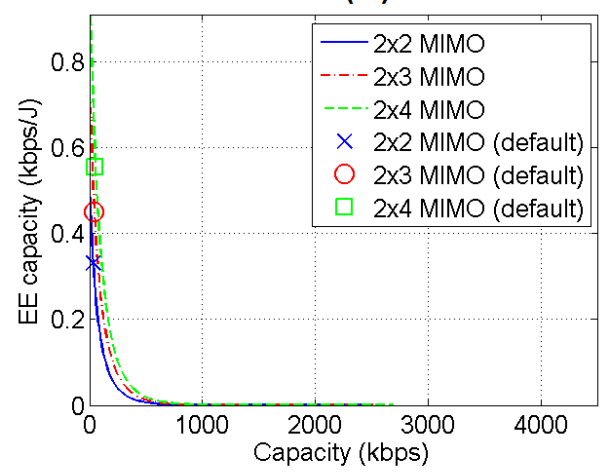

(d)

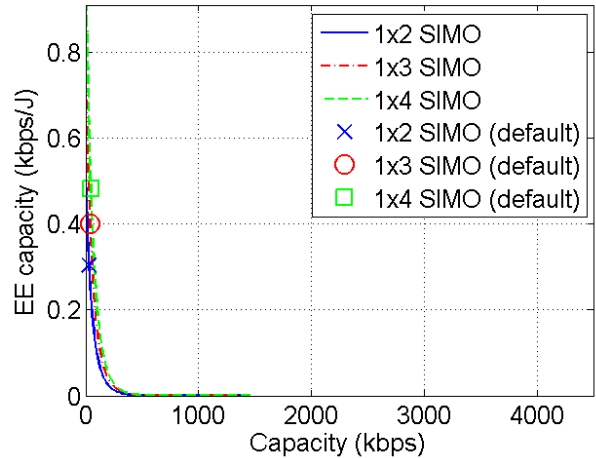

(b)

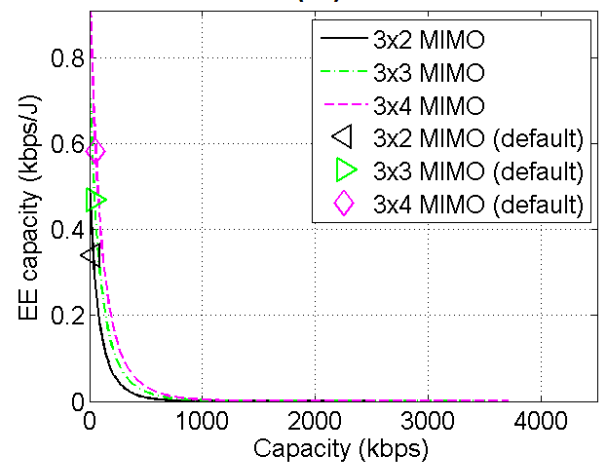

(e)

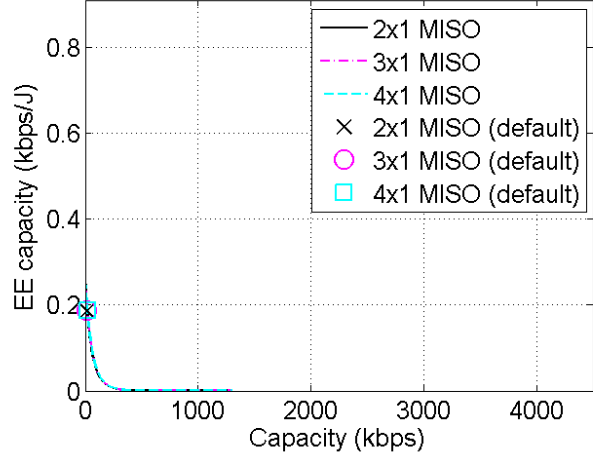

(c)

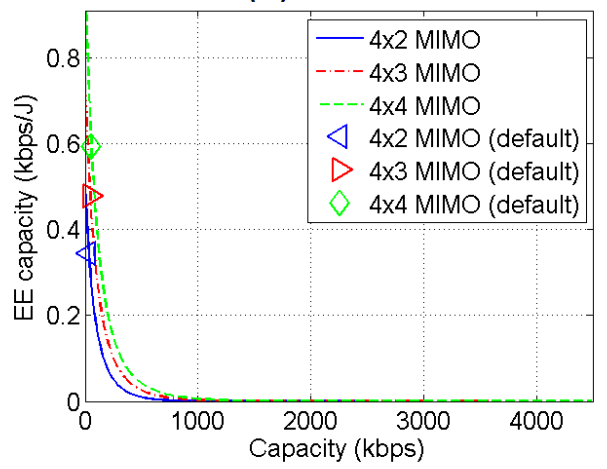

(f)

Fig. 6. Same curves with Figs(a)-(f) but for the power consumption scenario B.

- The concurrent SE and EE analysis clearly outlines the main deficiency of UWA networks deploying today's UWA system equipment; due to the inherent drawbacks of UWA channels, such as attenuation and noise, the SE and EE performance of UWA networks is significantly affected in comparison with other wireless and wireline broadband technologies [6], [59]. Although default IPSDM limits define a decent narrowband compromise both respecting capacity requirements and recent green technology considerations, the further broadband exploitation of UWA channels demands significantly higher IPSDM limits combined with more SE- and EE-aware UWA system equipment.

- Capacity differences of the order of hundreds of kbps are observed among the different IPSDM limits and multi-port schemes indicating how crucial for the future of broadband UWA technology in undersea IoT and M2M networks is the selection of suitable IPSDM limits and multi-port schemes. In fact, the UWA broadband perspective becomes meaningless when power injection is constrained by lower IPSDM limits (i.e., lower than the default IPSDM limits) even though their EE behavior seems to be excellent. Conversely, adopting high IPSDM limits (e.g. greater than $0 \mathrm{dBm} / \mathrm{Hz}$ ), all MIMO schemes present capacities that exceed $1 \mathrm{Mbps}$. However, in the case of high IPSDM limits, the applied EE metrics underline the poor EE performance of UWA networks and the mismanagement of the consumed power. Anyway, SE and EE metrics can be adjusted according to network capacity demands and ecological awareness. 
- Spectral capacity and power consumption are very sensitive to IPSDM limit changes. Through a slight reduction of IPSDM limits, better balance onto SE/EE trade-off curves may occur. Observing the right tail of $\mathrm{SE} / \mathrm{EE}$ trade-off curves, significant potential for power saving may occur without critically affecting UWA capacity.

- The significant channel attenuation of SISO/CCs and SISO/XCs presented in Figs. 3(a)-(d) affect the achievable SISO, SIMO and MISO capacities as well as their EE capacities. Simultaneously, as the cardinality of the active transmit and receive sets in SIMO and MISO schemes increases, $\mathrm{SE}$ and EE metrics get improved due to the increasing spatial multiplexing [10], [60]. Therefore, only SISO/CC, SISO/XC, $1 \times 4$ SIMO and $4 \times 1$ MISO configurations will be examined in the rest of the paper giving a representative upper bound of the respective schemes.

- The implementation of MIMO schemes mitigates the disappointing SE and EE picture of SISO, SIMO and MISO ones. For the same IPSDM limits, MIMO schemes simultaneously achieve better SE and EE metrics in comparison with SISO/CC, SISO/XC, SIMO and MIMO ones. Anyway, these results are in accordance with the traditional belief in other communications systems that MIMO schemes are more energy-efficient than SISO, MISO and SIMO ones [19], [48], [49], [54]. Hereafter, among different MIMO schemes, only $4 \times 4$ MIMO will be examined giving a representative upper bound of the UWA network performance.

- Comparing SE and EE performance of the two power consumption scenarios, their differences are negligible in all the cases examined. This is due to the fact that the main power consumption blocks in UWA transducers are their power amplifiers and, hence, the use of either traditional acoustic modems or software defined acoustic modems little affects the SE and EE performance as well as the proposed SE/EE trade-off curves. In the rest of this paper, only the general case of traditional acoustic modems (i.e., power consumption scenario A) is examined without affecting the validity of the following analysis.

- In UWA networks, the exact knowledge of SE/EE trade-off curves that implies the exact knowledge of swapping between IPSDM limits and MIMO schemes can define wiser green system design decisions. Adopting adaptive EMI policies, which can have adjustable IPSDM limits, significant SE and EE metric improvements can be achieved. Moreover, the impact on SE and EE capacity may further be mitigated or even be inversed, through the combined application of adaptive EMI policies, MIMO schemes and more EE UWA system equipment. As it has already been verified in [18], [59], the trade-off between capacity and EE capacity is a quasiconcave function. This SE/EE trade-off determines a dynamic equilibrium between the adopted IPSDM limits and the deployed MIMO configurations [59], [61]. However, SE/EE trade-off curves can be shifted when changes in noise environment and configuration parameters occur, hence, explaining the term of dynamic equilibrium. To investigate the behavior of SE/EE trade-off curves, the influence of different factors is studied on the basis of the following series of figures. Due to the significant EE capacity differences among different factors, the logarithmic scale on the EE capacity axis is applied, hereafter. 
More specifically, in Fig. 7(a), the proposed SE/EE trade-off between the median EE capacities of SISO/CC and 1x4 SIMO schemes and their respective median capacities is plotted for IPSDM limits ranging from $-100 \mathrm{dBm} / \mathrm{Hz}$ to $50 \mathrm{dBm} / \mathrm{Hz}$ with step $1 \mathrm{dBm} / \mathrm{Hz}$ when bad noise scenario (i.e., $-30 \mathrm{dBm} / \mathrm{Hz}$ ), average noise scenario (i.e., $-83 \mathrm{dBm} / \mathrm{Hz}$, default) and good noise scenario (i.e., $-120 \mathrm{dBm} / \mathrm{Hz}$ ) occur. The default IPSDM limits are denoted in all the cases examined. In Fig. 7(b), same curves are given with Fig. 7(a) but for the 4x1 MISO and 4x4 MIMO.

The noise variations, which are examined in Figs. 7(a) and 7(b), can be caused by several factors such as the motion of transmit and receive transducers, internal waves, surface waves, operation of nearby ship engines, changes in temperature, fish population, storms, changes in water depth, changes in water structure and weather conditions. Also, these noise variations may occur on various time scales such as seasonal cycles, diurnal cycles, tidal cycles, minutes and even seconds [15], [62]. This dynamic environment imposes significant difficulties during the determination of the noise conditions further complicating the analysis and design of UWA networks in undersea IoT and M2M environment.

To assess the impact of noise variations on the design of single- and multi-port UWA networks, suitable metrics such as capacity and EE capacity are again applied in Figs. 7(a) and 7(b). As it concerns SE performance of UWA networks, the capacity difference between good and bad noise scenario may reach up to $2.3 \mathrm{Mbps}, 2.3 \mathrm{Mbps}$, $2.3 \mathrm{Mbps}$ and $8.6 \mathrm{Mbps}$ for the examined SISO/CC, $1 \times 4 \mathrm{SIMO}, 4 \mathrm{x} 1 \mathrm{MISO}$ and $4 \times 4$ MIMO schemes, respectively. In all the cases examined, it should be noted that broadband UWA potential becomes meaningless when bad noise scenario is assumed even if high IPSDM limits and $4 \times 4$ MIMO arrangements are adopted. 

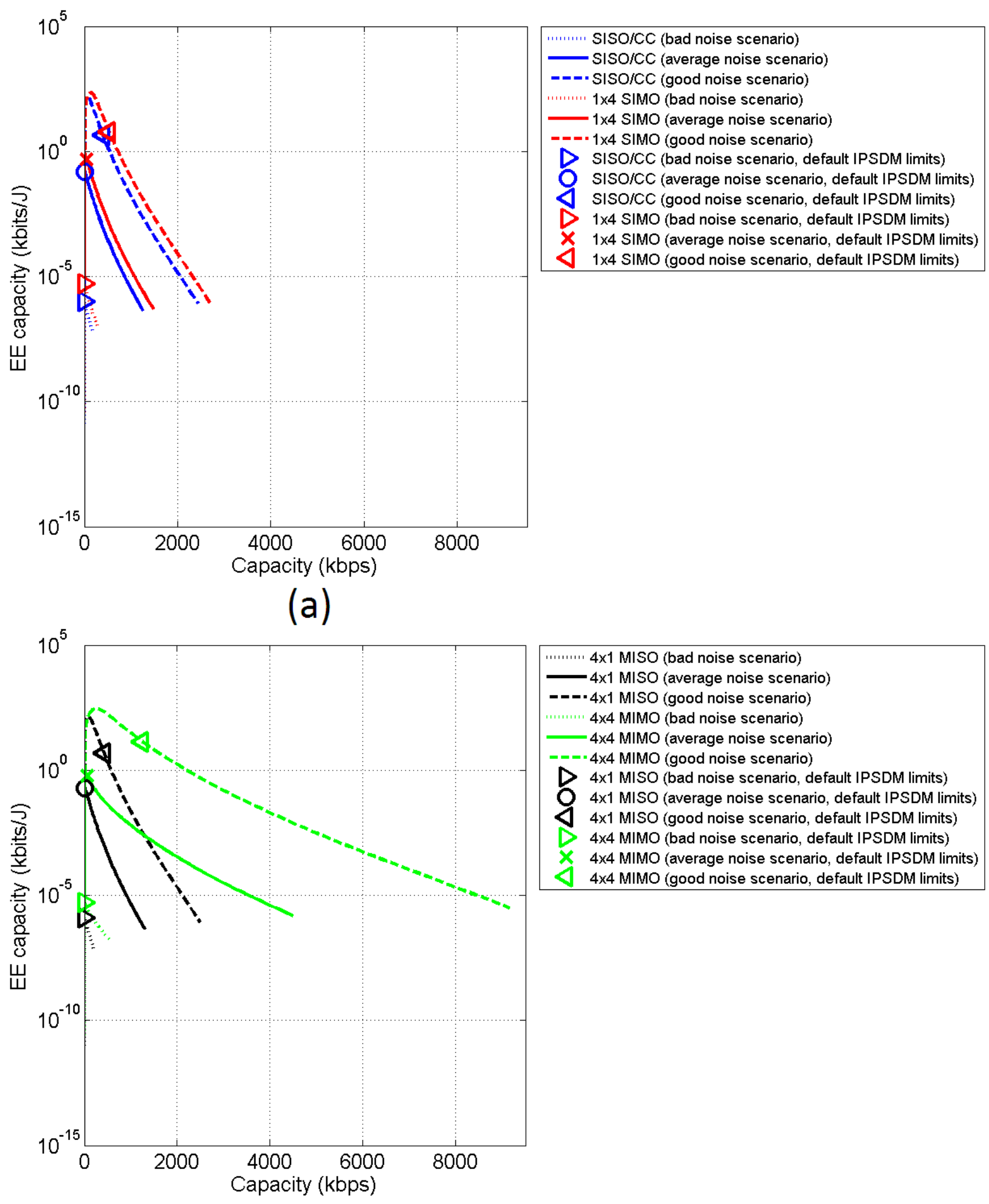

(b)

Fig. 7. Trade-off curves between median EE capacity and median capacity for various singleand multi-port schemes when different noise scenarios are applied (the logarithmic scale on $\mathrm{y}$-axis is used).

(a) SISO/CC and 1x4 SIMO trade-off curves. (b) 4x1 MISO and 4x4 MIMO trade-off curves. 
Similarly, significant EE capacity differences among the recently examined UWA networks are also observed. The EE capacity difference between good and bad noise scenario may reach up to $157 \mathrm{kbits} / \mathrm{J}, 220 \mathrm{kbits} / \mathrm{J}, 137 \mathrm{kbits} / \mathrm{J}$ and $284 \mathrm{kbits} / \mathrm{J}$ for the examined SISO/CC, 1x4 SIMO, 4x1 MISO and 4x4 MIMO schemes, respectively.

In fact, the default IPSDM limits offer a narrowband compromise between SE and EE performance. When default IPSDM limits are adopted, the capacity difference between good and bad noise scenario may reach up to $399 \mathrm{kbps}$, 536kbps, $425 \mathrm{kbps}$ and $1251 \mathrm{kbps}$ for the examined SISO/CC, $1 \mathrm{x} 4 \mathrm{SIMO}, 4 \mathrm{x} 1 \mathrm{MISO}$ and $4 \mathrm{x} 4$ MIMO schemes, respectively, whereas the EE capacity difference between good and bad noise scenario may reach up to $4.33 \mathrm{kbits} / \mathrm{J}, 5.81 \mathrm{kbits} / \mathrm{J}, 4.61 \mathrm{kbits} / \mathrm{J}$ and $13.54 \mathrm{kbits} / \mathrm{J}$ for the examined SISO/CC, $1 \times 4$ SIMO, 4x1 MISO and 4x4 MIMO schemes, respectively.

Anyway, the $4 \mathrm{x} 4 \mathrm{MIMO}$ arrangements that fully exploit the spatial diversity of UWA/MIMO configurations offer the best SE and EE results in comparison with the other applied schemes in all the cases examined. However, the weak point of the deployment of MIMO schemes is the high number of transducers that should be installed.

In Figs. 8(a) and 8(b), same curves with Figs. 7(a) and 7(b) are plotted when the default IPSDM limits and noise conditions are considered but for the three indicative UWA topologies -i.e., $z_{\mathrm{R} 1}=50 \mathrm{~m}, z_{\mathrm{R} 1}=100 \mathrm{~m}$ (default value) and $z_{\mathrm{R} 1}=1000 \mathrm{~m}-$.

Already highlighted in Figs. 4(a)-(c), the effect of distance among transmit and receive transducers on the SE and EE performance of UWA networks is also demonstrated in Figs. 8(a) and 8(b). In fact, attenuation of UWA channels becomes horrible for long-distance communication links, indicating that the dominant attenuation factor for this type of IoT and M2M applications is not longer the operating frequency but transmission losses. Even if high IPSDM limits and high cardinality MIMO schemes are adopted, the performance of long-range UWA channels remains anemic. This phenomenon is a classical feature that distinguishes a UWA channel from a terrestrial radio one [13]. 


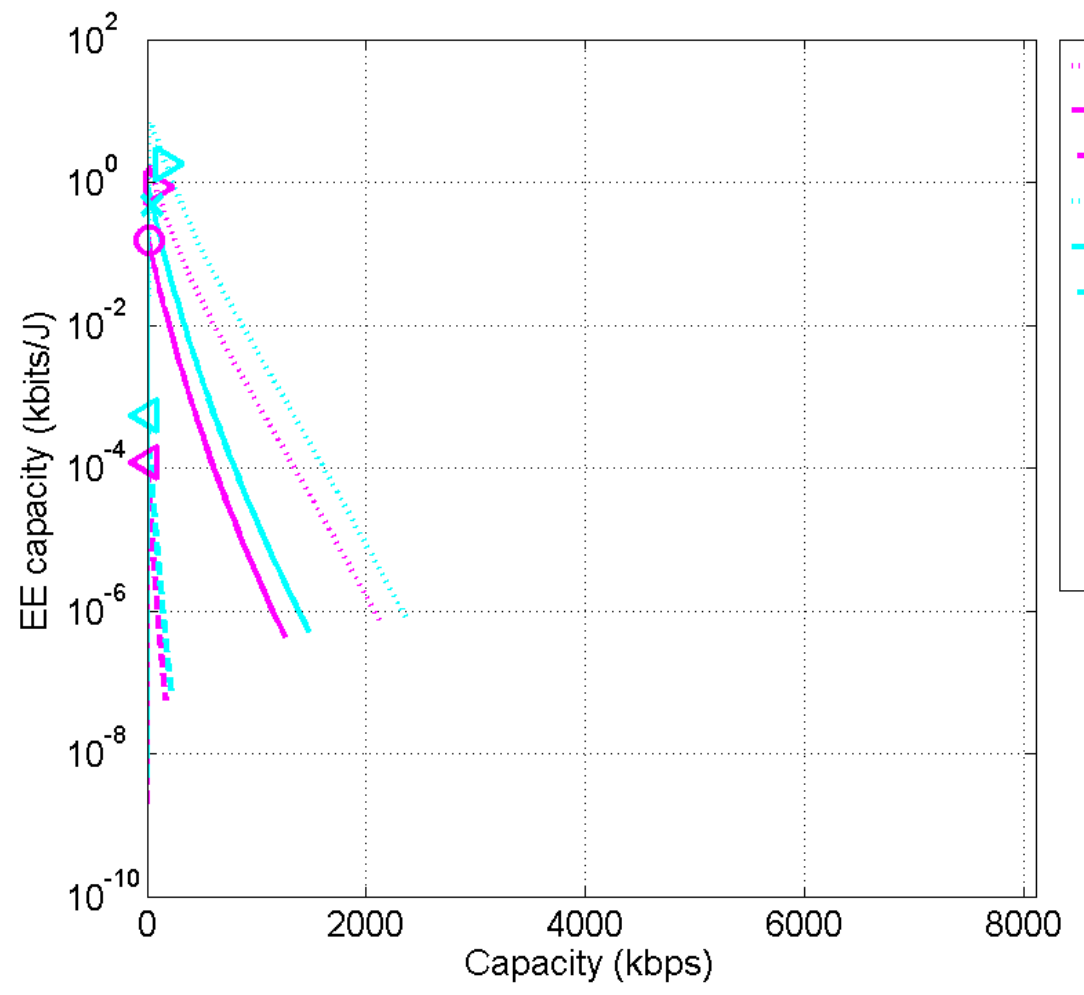

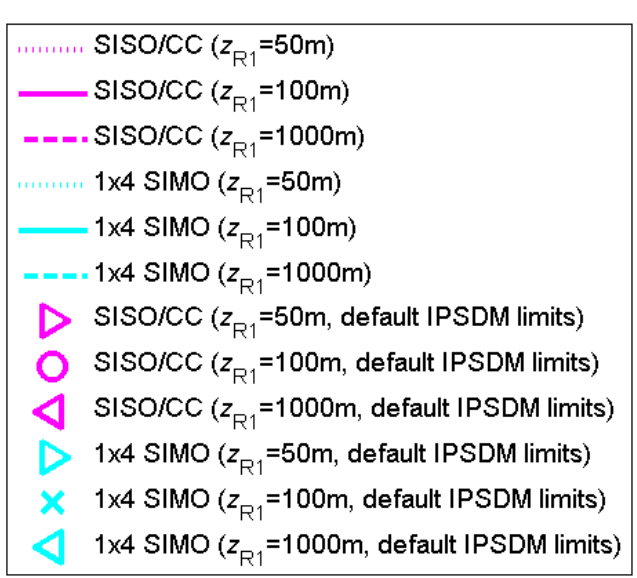

(a)

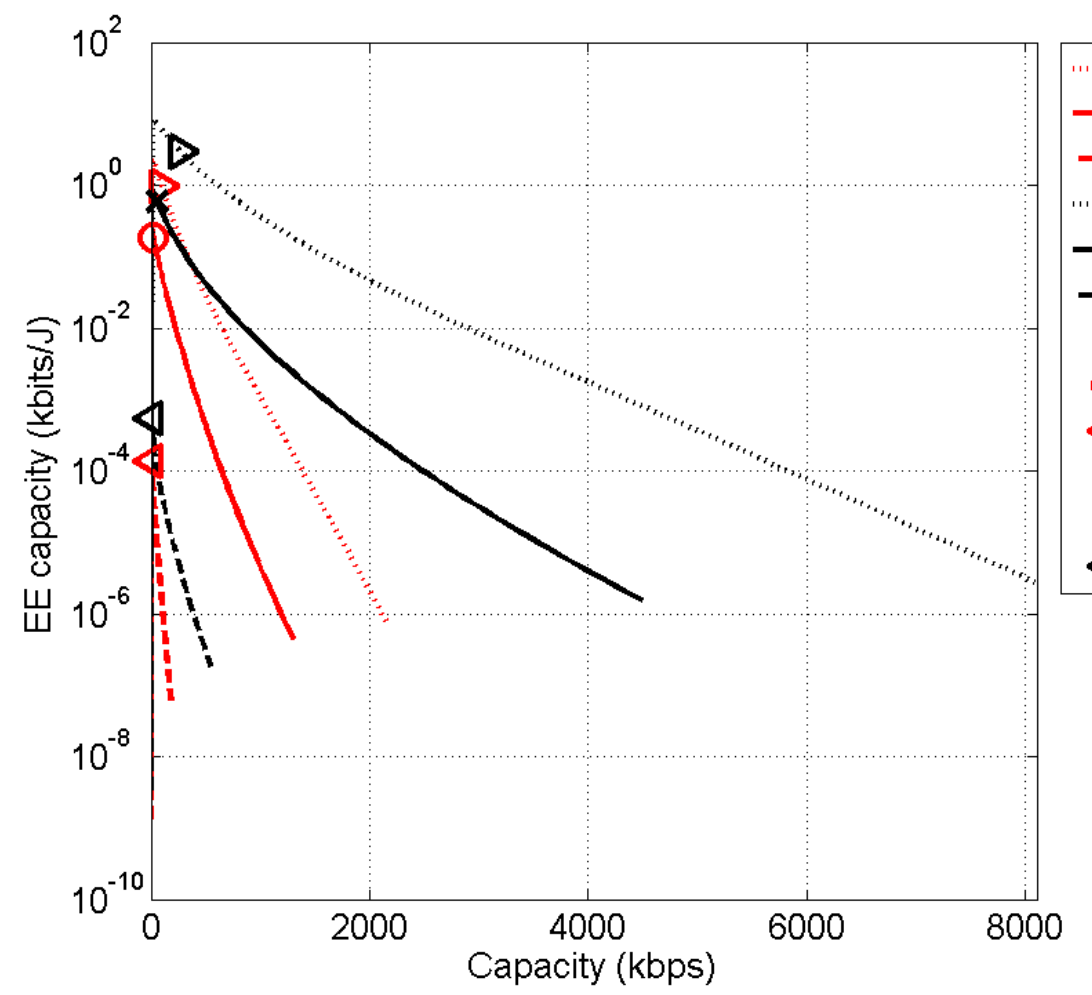

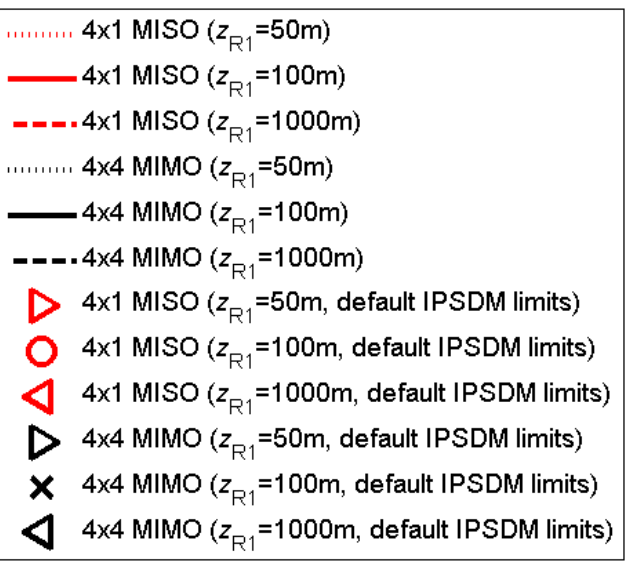

(b)

Fig. 8. Same curves with Figs. 7(a) and 7(b) but for default IPSDM limits and default noise conditions when different UWA topologies are examined. 
To deal with the destructive effect of distance, the solution lies in the proposal of two different architectures that are suitable for UWA networks. The first architecture is based on the deployment of distributed transmit/receive transducers communicating through a fixed infrastructure of transmit/receive transducers [63]. The fixed transmit/receive transducers act as base stations being mounted on surfaces buoys or being bottom mounted. The communication of base stations with the land communications networks is achieved either through wireless links or through wireline infrastructure depending on the UWA network position. The second architecture relies on decentralized ad-hoc networks without fixed infrastructure. Transmit/receive transducers communicate through multi-hop relaying of upper-bound maximum distance [7], [64]. Combining the findings of Figs. 8(a) and 8(b) with the aforementioned network architectures, high SE and EE performance can be secured in UWA networks when maximum distances between adjacent transmit/receive transducers remain shorter than a distance threshold.

Since the main idea of boosting SE and EE metrics of UWA networks is based on the deployment of high cardinality MIMO schemes and the adoption of standard topologies like those of the two aforementioned network architectures (e.g., topologies of maximum distance of $50 \mathrm{~m}$ or $100 \mathrm{~m}$ ), special attention should be given to the behavior of $4 \times 4$ MIMO scheme as well as the application of suitable IPSDM limits. Actually, when the default IPSDM limits are adopted, capacity and EE capacity of UWA/SISO networks of maximum distance of $50 \mathrm{~m}$ are equal to $78 \mathrm{kbps}$ and $0.84 \mathrm{kbits} / \mathrm{J}$, respectively, whereas the respective SE and EE metrics of UWA/SISO networks of maximum distance of $100 \mathrm{~m}$ are equal to $14 \mathrm{kbps}$ and $0.15 \mathrm{kbits} / \mathrm{J}$, respectively. In the case of the $4 \times 4 \mathrm{MIMO}$ scheme of maximum distance of $100 \mathrm{~m}$, the same capacity and EE capacity values with UWA/SISO networks of maximum distance of 50m are achieved when IPSDM limits are equal to approximately $-22 \mathrm{dBm} / \mathrm{Hz}$ and $-39 \mathrm{dBm} / \mathrm{Hz}$, respectively. Similarly, the same capacity and EE capacity values with UWA/SISO networks of maximum distance of $100 \mathrm{~m}$ are achieved when IPSDM limits are equal to approximately $-32 \mathrm{dBm} / \mathrm{Hz}$ and $-54 \mathrm{dBm} / \mathrm{Hz}$, respectively. It is evident that $100 \mathrm{~m}$-long MIMO schemes of high cardinality order combined with significantly lower IPSDM limits can efficiently substitute UWA/SISO networks of higher distances on the basis of the examined SE and EE metrics.

Except for the distance, significant role during the propagation of UWA signals plays the water depth that determines the richness of the occurred multipath environment. The influence of water depth on SE and EE performance of single- and multi-port UWA schemes is here examined. In Figs. 9(a) and 9(b), same curves with Figs. 7(a) and 7(b) are drawn when the default IPSDM limits and noise conditions are applied but for three different water depths - i.e., $D=2.4 \mathrm{~m}, D=100 \mathrm{~m}$ (default value) and $D=1000 \mathrm{~m}-$. Although the last value of water depth lies outside the assumption of a surrounding shallow water environment, it provides the SE/EE trade-off curve trend.

From Figs. 9(a) and 9(b), it is evident that as the water depth increases the SE and EE capacity of MIMO schemes slowly decrease. However, below a certain depth threshold (e.g., $100 \mathrm{~m}$ ), the performance deterioration remains marginal. Conversely, the best performance of single- and multi-port schemes is achieved in very shallow waters (i.e., rivers, beaches, lakes, etc). This is explained by the fact that 

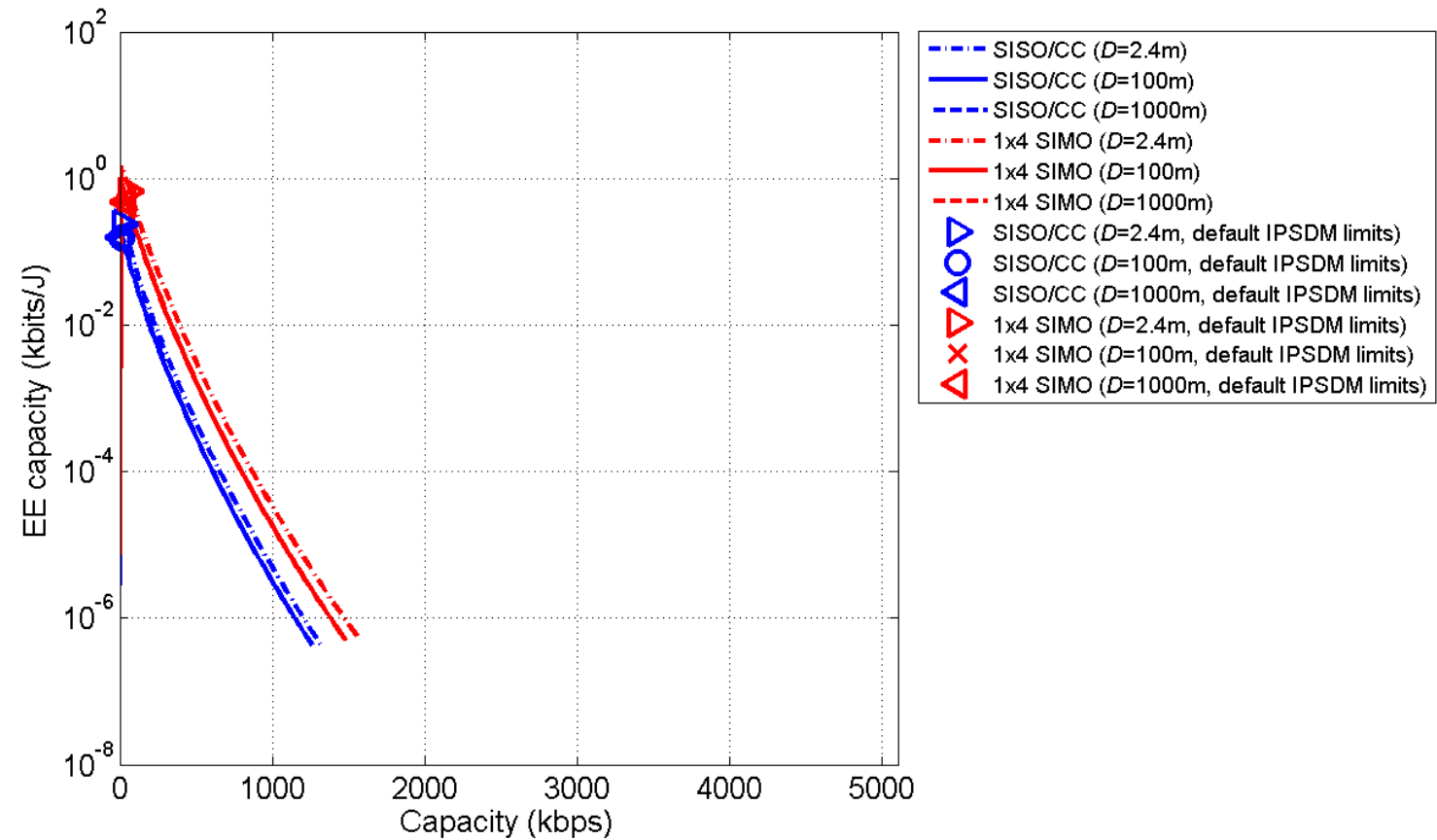

(a)
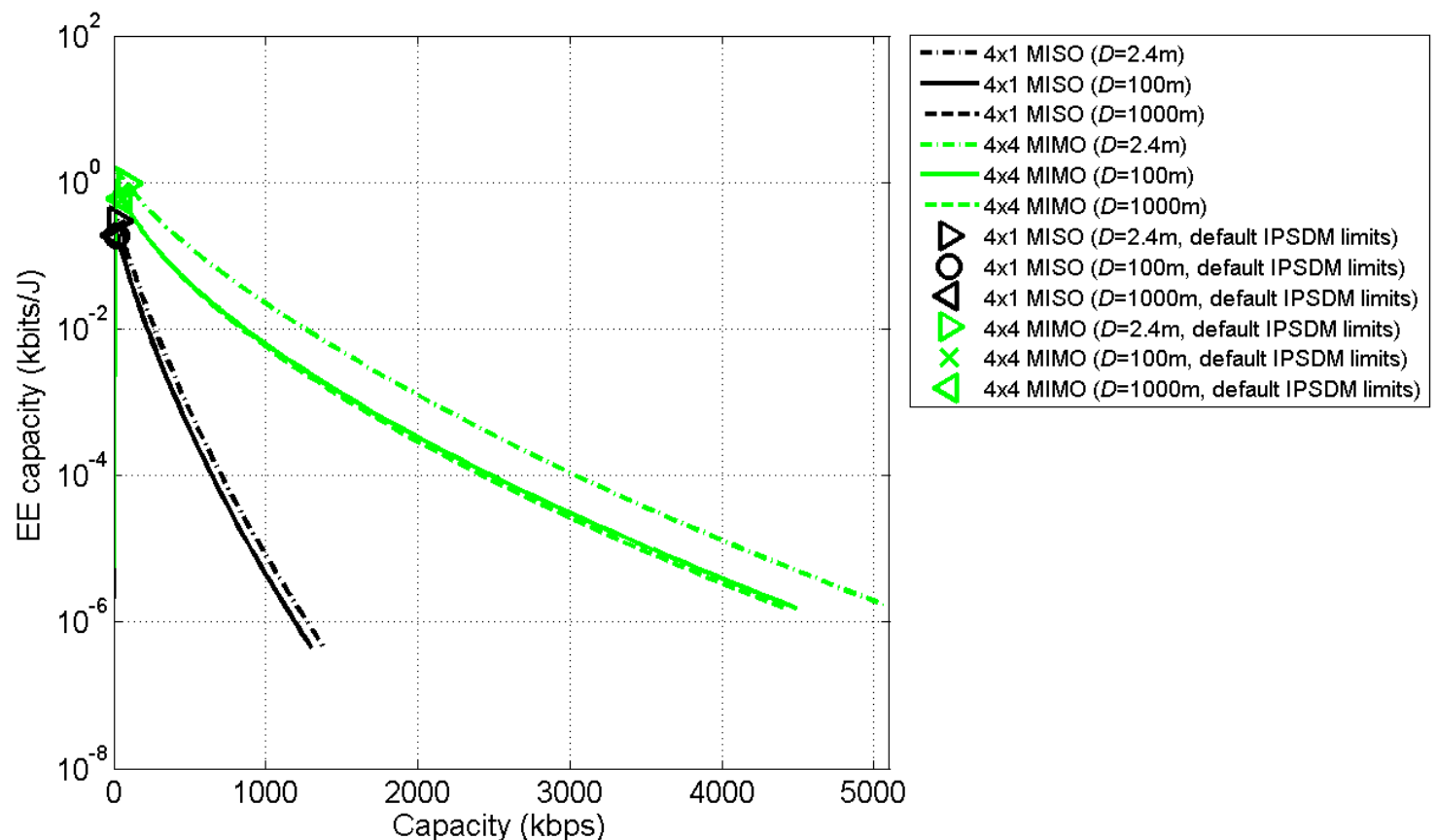

(b)

Fig. 9. Same curves with Figs. 7(a) and 7(b) but for default IPSDM limits and default noise conditions when different water depths are examined.

capacity of UWA networks strongly depends on the correlation among different $\mathrm{SISO} / \mathrm{CC}$ and SISO/XC channels mathematically represented by the sum term of eqs. 
(26)-(28). In accordance with [13], the lower is the spatial correlation the larger is the MIMO gain implying better SE and EE capacities. The lowest spatial correlation is reached in the case of rich multi-paths environments that are present when channel water depth is very shallow (i.e., near the shores) [13].

Finally, apart from the depth, the factor of spatial correlation, which is strongly related to the spatial multiplexing and SE/EE performance of single- and multi-port schemes, is influenced by the spacings of transmit and receive transducers. In order to study the influence of spacings, in Figs. 10(a) and 10(b), same curves with Figs. 7(a) and 7(b) are drawn when the default IPSDM limits and default noise conditions are applied but for three different vertical spacings of transmit transducers -i.e., $\Delta_{\mathrm{yT}}=0 \mathrm{~m}, \Delta_{\mathrm{yT}}=0.6 \mathrm{~m}$ (default value) and $\Delta_{\mathrm{yT}}=6 \mathrm{~m}-$. In Figs. 10(c) and 10(d), same curves are given with Figs. 10(a) and 10(b) but for three different vertical spacings of receive transducers -i.e., $\Delta_{\mathrm{yR}}=0 \mathrm{~m}, \Delta_{\mathrm{yR}}=0.6 \mathrm{~m}$ (default value) and $\Delta_{\mathrm{yR}}=6 \mathrm{~m}-$.

Observing the previous Figs. 10(a)-(d), interesting conclusions may be deduced:

- As it concerns the transducer spacings, the behavior of different schemes varies depending on the scheme type (i.e., SISO/CC, SIMO, MISO and MIMO one), the spacing type (i.e., transmit or receive transducer spacing) and the spacing value. More specifically:

- In the case of $\mathrm{SISO} / \mathrm{CC}$ networks, capacity initially improves with increasing transducer spacing regardless of the spacing type. Beyond a transducer spacing threshold, capacity drops off while increasing transducer spacing. This is due to the fact that the spacing increase implies distance increase that further aggravates SISO channel attenuation and respective capacities. This transducer spacing threshold depends on the distance among transmit and receive transducers and the UWA configuration.

- In the case of SIMO networks, the different spacing types have different impact on SE and EE metrics of these networks. As it concerns the transmit transducer spacing, this change has negligible effect on the median channel attenuation of SIMO schemes since the median distance between transmit transducer and the receive transducers remains almost 


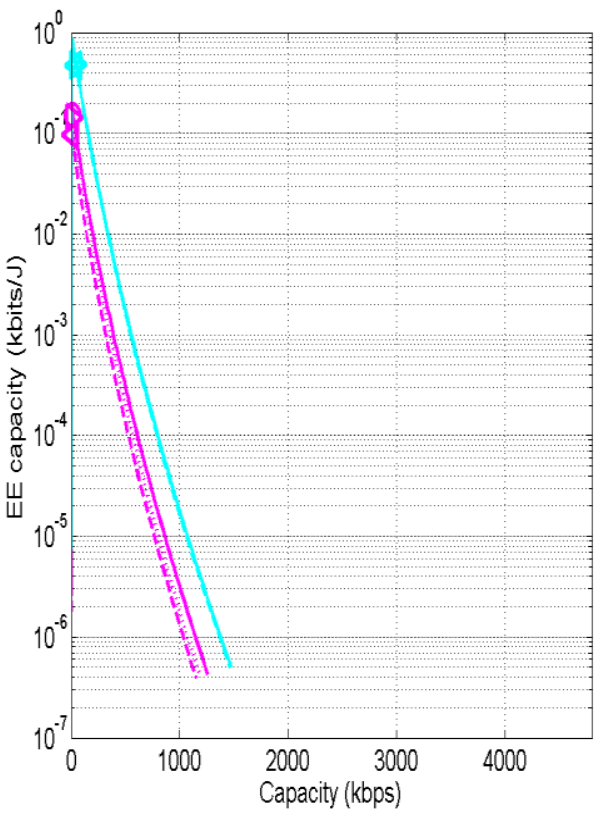

(a)

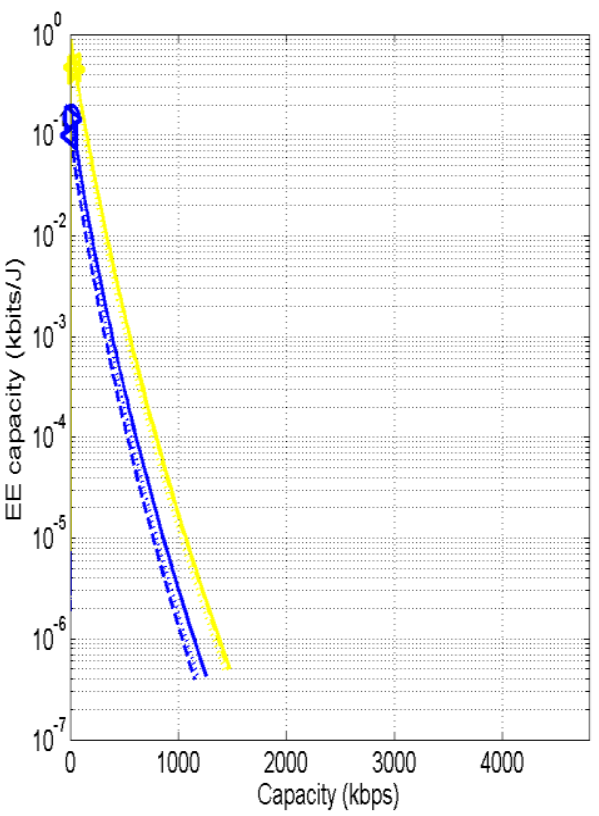

(c)
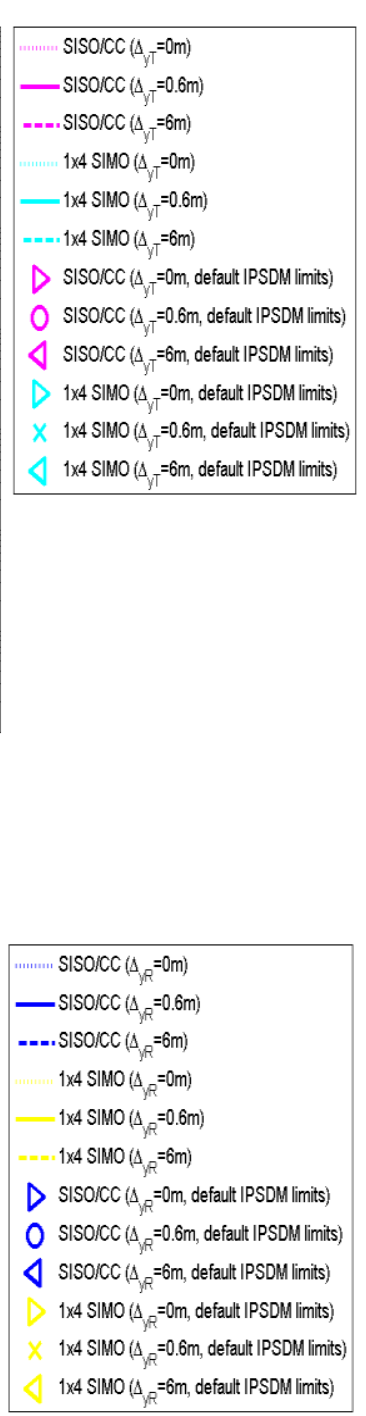

Fig. 10. Same curves with Figs. 7(a) and 7(b) but for default IPSDM limits and default noise conditions when different transducer spacings occur. (a) Different vertical spacings of transmit transducers: SISO/CC and 1x4 SIMO trade-off curves. (b) Different vertical spacings of transmit

transducers: 4x1 MISO and 4x4 MIMO trade-off curves. (c) Different vertical spacings of receive transducers: SISO/CC and 1x4 SIMO trade-off curves. (d) Different vertical spacings of receive transducers: $4 \times 1$ MISO and 4x4 MIMO trade-off curves.

stable. Thus, the increase of transmit transducer spacing little affects the capacity of SIMO networks. In contrast with transmit transducer spacing, 
the increase of receive transducer spacing slightly deteriorates the SE performance of SIMO networks. On the basis of the receive transducer spacing impact on SISO/CC networks, the spatial multiplexing of SIMO networks achieves to mitigate the arisen capacity reductions during the increase of receive transducer spacing.

- Similarly to SIMO networks, in the case of MISO ones, the different spacing types differently influence SE metrics of these networks. However, due to the architecture similarities between SIMO and MISO networks, the influence of transmit transducer spacing on the capacity of MISO networks is the same with the influence of receive transducer spacing of SIMO networks. Similar SE performance results occur during the changes in the receive transducer spacing of MISO networks with the transmit transducer spacing of SIMO networks.

- In the case of MIMO networks, the changes of transducer spacing have totally different effect on capacity in comparison with the respective changes in SISO/CC, SIMO and MISO networks. Actually, the exploitation of spatial multiplexing in UWA/MIMO networks is so intense that the effect of distance increase on SE metrics remains limited. Nevertheless, above a certain transducer spacing threshold, the improvement of capacity becomes marginal. To further boost the SE performance of MIMO networks, MIMO schemes of higher cardinality should be deployed.

- With reference to eq. (31), EE capacity depends on the capacity and power consumption. Since power consumption remains stable as transducer spacing changes, EE capacity present the same behavior with capacity during the previous transducer spacing changes in all examined single- and multi-port schemes. Mathematically, the impact percentage of transducer spacing changes on median capacity and median EE capacity is analytically reported in Table 4 where the previous observations are verified.

\section{Road Map for the UWA Future Research in loT and M2M Landscape}

First, apart from the SE and EE performance of UWA networks, crucial matter for their further development is their interoperability potential with other already licensed broadband technologies in IoT and M2M environment. The latter technologies are essential for the connectivity of UWA networks with the land communications systems. However, before UWA networks coexist with these broadband technologies -wired, such as fiber and DSL, and wireless, such as WiFi and WiMax-, the UWA technology intraoperability needs to be further exploited. Apart from compatible frequencies, equipment signaling, UWA network standardization, wise design of UWA configurations, adoption of MIMO technology and the promotion of the concepts of scalable capacity and standard topologies, green issues should be readdressed taking into account the today's poor SE and EE performance of UWA networks.

Second, extending the applicability and practicability of SE/EE trade-off curves to real UWA/MIMO networks, throughput can be used instead of capacity. Since the future research will focus on real UWA networks, the influence of the adopted modulations and coding schemes on the throughput reduction should be investigated studying the respective SE/EE trade-off curves either in shape or in maxima [65], [66]. Anyway, the application of SE/EE trade-off curves in real single- and multi-port 
UWA networks using more sophisticated: (i) channel approximation techniques; and (ii) RF MIMO architectures, modulations and applications inspired by other wireless MIMO communications networks [67]-[70]; is going to be further analyzed in the oncoming research works.

TABLE IV

The Influence of Transducer Spacing on Median Capacity and Median EE Capacity for Different Single- and Multi-Port Schemes

(Green color: increase; Red color: decrease)

\begin{tabular}{|c|c|c|c|c|c|c|}
\hline $\begin{array}{l}\text { MIMO } \\
\text { scheme }\end{array}$ & $\begin{array}{l}\text { Change of } \\
\text { Transmit } \\
\text { Transducer } \\
\text { Spacing }\end{array}$ & $\begin{array}{c}\text { Capacity } \\
\text { Percentage } \\
\text { Change }\end{array}$ & $\begin{array}{c}\text { EE } \\
\text { Capacity } \\
\text { Percentage } \\
\text { Change }\end{array}$ & $\begin{array}{l}\text { Change of } \\
\text { Receive } \\
\text { Transducer } \\
\text { Spacing }\end{array}$ & $\begin{array}{c}\text { Capacity } \\
\text { Percentage } \\
\text { Change }\end{array}$ & $\begin{array}{c}\text { EE } \\
\text { Capacity } \\
\text { Percentage } \\
\text { Change }\end{array}$ \\
\hline \multirow[t]{2}{*}{$\mathrm{SISO} / \mathrm{CC}$} & $0 \mathrm{~m} \rightarrow 0.6 \mathrm{~m}$ & $8.40 \%$ & $8.40 \%$ & $0 \mathrm{~m} \rightarrow 0.6 \mathrm{~m}$ & $9.45 \%$ & $9.45 \%$ \\
\hline & $0 \mathrm{~m} \rightarrow 6 \mathrm{~m}$ & $-33.08 \%$ & $-33.08 \%$ & $0 \mathrm{~m} \rightarrow 6 \mathrm{~m}$ & $-29.68 \%$ & $-29.68 \%$ \\
\hline \multirow{2}{*}{$\begin{array}{c}\text { SIMO } \\
1 \times 4\end{array}$} & $0 \mathrm{~m} \rightarrow 0.6 \mathrm{~m}$ & $-0.09 \%$ & $-0.09 \%$ & $0 \mathrm{~m} \rightarrow 0.6 \mathrm{~m}$ & $3.98 \%$ & $3.98 \%$ \\
\hline & $0 \mathrm{~m} \rightarrow 6 \mathrm{~m}$ & $-2.3 \%$ & $-2.3 \%$ & $0 \mathrm{~m} \rightarrow 6 \mathrm{~m}$ & $3.05 \%$ & $3.05 \%$ \\
\hline \multirow{2}{*}{$\begin{array}{c}\text { MISO } \\
4 \times 1 \\
\end{array}$} & $0 \mathrm{~m} \rightarrow 0.6 \mathrm{~m}$ & $3.99 \%$ & $3.99 \%$ & $0 \mathrm{~m} \rightarrow 0.6 \mathrm{~m}$ & $-0.05 \%$ & $-0.05 \%$ \\
\hline & $0 \mathrm{~m} \rightarrow 6 \mathrm{~m}$ & $3.05 \%$ & $3.05 \%$ & $0 \mathrm{~m} \rightarrow 6 \mathrm{~m}$ & $-2.89 \%$ & $-2.89 \%$ \\
\hline \multirow{2}{*}{$\begin{array}{c}\text { MIMO } \\
4 \times 4\end{array}$} & $0 \mathrm{~m} \rightarrow 0.6 \mathrm{~m}$ & $23.02 \%$ & $23.02 \%$ & $0 \mathrm{~m} \rightarrow 0.6 \mathrm{~m}$ & $23.02 \%$ & $23.02 \%$ \\
\hline & $0 \mathrm{~m} \rightarrow 6 \mathrm{~m}$ & $33.36 \%$ & $33.36 \%$ & $0 \mathrm{~m} \rightarrow 6 \mathrm{~m}$ & $33.41 \%$ & $33.41 \%$ \\
\hline
\end{tabular}

Third, the combined operation of UWA networks with other supported communications networks in the IoT and M2M framework can also significantly improve the SE/EE trade-off curves of UWA networks at a local basis as well as the insertion of new metrics from other already established communications technologies during the performance analysis of undersea communications [71]-[74]. Hence, the development of new ad-hoc trade-off curves of UWA networks at a local and daily basis and the stabilization of these trade-off curves when various fluctuations occur in UWA surrounding environment and UWA modem equipment define another two critical UWA research topics.

\section{Conclusions}

This paper has focused on the SE and EE performance of single- and multi-port UWA networks in the $0-100 \mathrm{kHz}$ frequency range. Their performance has been investigated with respect to: (i) different single- and multi-port schemes and configurations; (ii) different IPSDM limits and various noise conditions; (iii) different power consumption scenarios due to UWA acoustic modem equipment; (iv) new transmission, SE and EE metrics; (v) EE communications principles; and (vi) the proposed SE/EE trade-off curves.

Based on the applied SE and EE metrics as well as the proposed SE/EE trade-off curves, major features of UWA networks have been reviewed for use in future's practical UWA networks. Information theory has revealed that capacity of UWA/MIMO networks can reach up to $10 \mathrm{Mbps}$ when high IPSDM limits, full MIMO schemes and short- and medium-range communications links are adopted regardless of the UWA acoustic modem equipment. To practically achieve these high data rates in the $0-100 \mathrm{kHz}$ frequency band, the concepts of multi-hop UWA communications and standard UWA topologies has been promoted in this paper. At the same time, 
critical role towards higher SE performances of UWA networks plays the wise design of multi-port arrangements and configurations in accordance with SE/EE trade-off curves. Maladjustments of MIMO configuration properties such as: (i) distance among transmit and receive transducers; and (ii) horizontal and vertical spacing of transmit and receive transducers; may create Mbps of capacity differences.

As it concerns the EE properties of UWA networks, the main conclusion is that today's transducer technology does not permit the coexistence of the full broadband exploitation of UWA networks along with their EE operation. Even if full MIMO schemes are applied and software defined acoustic modems are deployed, the capacity of UWA networks is constrained far below the broadband capacity threshold of $2-3 \mathrm{Mbps}$. Therefore, till new more EE UWA equipment is available, a strategic decision concerning the further development of UWA networks should be made: EE UWA networks of few kbps data rates or non-EE UWA networks with broadband potential? The right answer depends on the throughput requirements of the projected applications while exploiting the quasiconcave SE/EE trade-offs that determine dynamic equilibria between capacity and EE capacity. Anyway, a better compromise between capacity performance and power consumption may occur in the following years.

Finally, this paper has introduced an important first step towards the design/operation of faster and greener UWA/IoT and UWA/M2M networks that are a communications world of cooperation. Based on new practical SE/EE trade-off curves in terms of throughput, the second step is going to cope with UWA/MIMO networks of real life.

\section{Conflicts of Interest}

The author declares that there is no conflict of interests regarding the publication of this paper.

\section{References}

[1] M. C. Domingo, "An overview of the internet of underwater things," Journal of Network and Computer Applications, vol. 35, no. 6, pp. 1879-1890, 2012. DOI: 10.1016/j.jnca.2012.07.012

[2] M. Zorzi, A. Gluhak, S. Lange, and A. Bassi, "From today's intranet of things to a future internet of things: a wireless-and mobility-related view," IEEE Wireless Communications, vol. 17, no. 6, pp. 44-51, 2010. DOI: 10.1109/MWC.2010.5675777

[3] R. Roman, C. Alcaraz, J. Lopez, and N. Sklavos, "Key management systems for sensor networks in the context of the Internet of Things," Computers \& Electrical Engineering, vol. 37, no. 2, pp. 147-159, 2011. DOI: 10.1016/j.compeleceng.2011.01.009

[4] H. P. Tan, R. Diamant, W. K. Seah, and M. Waldmeyer, "A survey of techniques and challenges in underwater localization," Ocean Engineering, vol. 38, no. 14, pp. 1663-1676, 2011. DOI: 10.1016/j.oceaneng.2011.07.017

[5] S. Arnon, "Underwater optical wireless communication network," Optical Engineering, vol. 49, no.1, pp. 1-6, 2010. DOI: 10.1117/1.3280288

[6] C. Yan, Z. Shunqing, X. Shugong, and G. Y. Li, "Fundamental trade-offs on green wireless networks," IEEE Commun. Mag., vol. 49, no. 6, pp. 30-37, Jun. 2011. DOI: 10.1109/MCOM.2011.5783982 
[7] A. Stefanov and M. Stojanovic, "Design and performance analysis of underwater acoustic networks," IEEE Journal on Selected Areas in Communications, vol. 29, no. 10, pp. 2012-2021, 2011. DOI: 10.1109/JSAC.2011.111211

[8] M. Stojanovic, "Underwater Acoustic Communications: Design Considerations on the Physical Layer," IEEE/IFIP Fifth Annual Conference on Wireless On demand Network Systems and Services (WONS 2008), Garmisch-Partenkirchen, Germany, Jan. 2008. DOI: 10.1109/WONS.2008.4459349

[9] A. Radosevic, J. G. Proakis, and M. Stojanovic, "Statistical characterization and capacity of shallow water acoustic channels," in IEEE OCEANS 2009-EUROPE, pp. 1-8, May 2009. DOI: 10.1109/OCEANSE.2009.5278349

[10] M. Zatman and B. Tracey, "Underwater acoustic MIMO channel capacity," in IEEE Conference Record of the Thirty-Sixth Asilomar Conference on Signals, Systems and Computers, vol. 2, pp. 1364-1368, Nov. 2002. DOI: 10.1109/ACSSC.2002.1197002

[11] R. F. Ormondroyd, "A robust underwater acoustic communication system using OFDM-MIMO,” IEEE OCEANS 2007-Europe, pp. 1-6, 2007. DOI: 10.1109/OCEANSE.2007.4302422

[12] A. G. Zajic, "Statistical modeling of MIMO mobile-to-mobile underwater channels," IEEE Transactions on Vehicular Technology, pp. 1337-1351, vol. 60, no. 4, 2011. DOI: 10.1109/TVT.2011.2129603

[13] P. Bouvet and A. Loussert, "Capacity analysis of underwater acoustic MIMO communications," IEEE OCEANS 2010, Sydney, 2010. DOI: 10.1109/OCEANSSYD.2010.5603661

[14] R. F. W. Coates, Underwater Acoustic Systems. Wiley, 1989.

[15] P. A. van Walree, "Propagation and Scattering Effects in Underwater Acoustic Communication Channels," IEEE Journal of Oceanic Engineering, vol. 38, no. 4, pp. 614-631, Oct. 2013. DOI: 10.1109/JOE.2013.2278913

[16] F. Guerra, P. Casari, and M. Zorzi, "World ocean simulation system (WOSS): A simulation tool for underwater networks with realistic propagation modeling," in Proc. ACM WUWNet, Berkeley, CA, USA, Nov. 2009, DOI: 10.1145/1654130.1654134.

[17] N. Parrish, L. Tracy, S. Roy, P. Arabshahi, and W. L. J. Fox, "System design considerations for undersea networks: Link and multiple access protocols," IEEE J. Sel. Areas Commun., vol. 26, no. 9, pp. 1720-1730, Dec. 2008. DOI: 10.1109/JSAC.2008.081211

[18] A. G. Lazaropoulos, "Overhead and Underground MIMO Low Voltage Broadband over Power Lines Networks and EMI Regulations: Towards Greener Capacity Performances," Computers and Electrical Engineering, vol. 39, pp. 2214-2230, 2013. DOI: 10.1016/j.compeleceng.2013.02.003

[19] A. G. Lazaropoulos, "Green Overhead and Underground Multiple-Input MultipleOutput Medium Voltage Broadband over Power Lines Networks: EnergyEfficient Power Control," Journal of Global Optimization, vol. 2012 / Print ISSN 0925-5001, pp. 1-28, Oct. 2012. DOI: 10.1007/s10898-012-9988-y

[20] A. Radosevic, D. Fertonani, T. M. Duman, J. G. Proakis, and M. Stojanovic, "Capacity of MIMO systems in shallow water acoustic channels," In IEEE 2010 Conference Record of the Forty Fourth Asilomar Conference on Signals, Systems and Computers (ASILOMAR), pp. 2164-2168, Nov. 2010. DOI:10.1109/ACSSC.2010.5757934 
[21] C. Gabriel, M. A. Khalighi, S. Bourennane, P. Léon, and V. Rigaud, "MonteCarlo-based channel characterization for underwater optical communication systems," Journal of Optical Communications and Networking, vol. 5, no. 1, pp. 1-12, 2013. DOI: 10.1364/JOCN.5.000001

[22] H. Dol, M. A. Ainslie, M. Colin, and J. Janmaat, "Simulation of an underwater acoustic communication channel characterized by wind-generated surface waves and bubbles," IEEE Journal of Oceanic Engineering, vol. 38, no. 4, pp. 642-654, Oct. 2013. DOI: 10.1109/JOE.2013.2278931

[23] E. Y. T. Kuo, "Sea surface scattering and propagation loss: Review, update and new predictions," IEEE J. Ocean. Eng., vol. OE-13, no. 4, pp. 229-234, Oct. 1988. DOI: $10.1109 / 48.9235$

[24] D. E. Weston and P. A. Ching, "Wind effects in shallow-water acoustic transmission,” J. Acoust. Soc. Amer., vol. 86, no. 4, pp. 1530-1545, Oct. 1989. DOI: $10.1121 / 1.398713$

[25] H. DeFerrari, N. Williams, and H. Nguyen, "Focused arrivals in shallow water propagation in the Straits of Florida," ARLO, vol. 4, no. 3, pp. 106-111, Jul. 2003. DOI: $10.1121 / 1.1591713$

[26] J. W. Choi and P. H. Dahl, "Mid-to-high-frequency bottom loss in the East China Sea," IEEE J. Ocean. Eng., vol. 29, no. 4, pp. 980-987, Oct. 2004. DOI: 10.1109/JOE.2004.834178

[27] O. Diachok, "Effects of absorptivity due to fish on transmission loss in shallow water," J. Acoust. Soc. Amer., vol. 105, no. 4, pp. 2107-2128, Apr. 1999. DOI: $10.1121 / 1.426816$

[28] J. M. Jornet, M. Stojanovic, and M. Zorzi, "On joint frequency and power allocation in a cross-layer protocol for underwater acoustic networks," IEEE $J$. Ocean. Eng., vol. 35, no. 4, pp. 936-947, Oct. 2010. DOI: 10.1109/JOE.2010.2080410

[29] D. Pompili, T. Melodia, and I. F. Akyildiz, "A CDMA-based medium access control for underwater acoustic sensor networks," IEEE Trans. Wireless Commun., vol. 8, no. 4, pp. 1899-1909, Apr. 2009. DOI: 10.1109/TWC.2009.080195

[30] M. A. Chitre, "A high-frequency warm shallow water acoustic communications channel model and measurements," Journal of the Acoustical Society of America, vol. 5, no. 122, pp. 2580-2586, 2007. DOI: 10.1121/1.2782884.

[31] X. Geng and A. Zielinski, "An eigenpath underwater acoustic communication channel model," in Proceedings of OCEAN'95, vol. 2, 1995, pp. 1189-1196. DOI: 10.1109/OCEANS.1995.528591

[32] C. S. Clay and H. Medwin, Acoustical Oceanography: Principles and Applications. New York: Wiley, 1977, ch. 3.

[33] W. H. Thorp, "Analytic description of the low frequency attenuation coefficient," Journal of the Acoustical Society of America, vol. 33, pp.334-340, 1961.

[34] F. H. Fisher and V. P. Simons, "Sound absorption in seawater," Journal of the Acoustical Society of America, vol. 62, pp. 558-564, 1977. DOI: 10.1121/1.381574

[35] D. Schneider, A. Schwager, J. Speidel and A. Dilly, "Implementation and Results of a MIMO PLC Feasibility Study," in Proc. IEEE Int. Symp. Power Line Communications and Its Applications, Udine, Italy, Apr. 2011, pp. 54-59. DOI: 10.1109/ISPLC.2011.5764450 
[36] E. Biglieri, J. Proakis, and S. Shamai (Shitz), "Fading channels: Information theoretic and communications aspects," IEEE Trans. Inform. Theory, vol. 44, pp. 2619-2692, Oct. 1998. DOI: 10.1109/18.720551

[37] A. Canova, N. Benvenuto, and P. Bisaglia, "Receivers for MIMO-PLC channels: Throughput comparison," in Proc. IEEE Int. Symp. Power Line Communications and Its Applications, Rio de Janeiro, Brazil, Mar. 2010, pp. 114-119. DOI: 10.1109/ISPLC.2010.5479904

[38] R. Hashmat, P. Pagani, A. Zeddam, and T. Chonavel, "MIMO communications for inhome PLC networks: Measurements and results up to $100 \mathrm{MHz}$," in Proc. IEEE Int. Symp. Power Line Communications and Its Applications, Rio de Janeiro, Brazil, Mar. 2010, pp. 120-124. DOI: 10.1109/ISPLC.2010.5479897

[39] D. Schneider, J. Speidel, L. Stadelmeier, and D. Schill, "Precoded spatial multiplexing MIMO for inhome power line communications," in Proc. IEEE Global Telecommunications Conference, New Orleans, LA, USA, Nov./Dec. 2008, pp. 1-5. DOI: 10.1109/GLOCOM.2008.ECP.556

[40] R. S. Prabhu and B. Daneshrad, "Energy-efficient power loading for a MIMOSVD system and its performance in flat fading," in Proc. IEEE Global Telecommunications Conference, Miami, FL, USA, Dec. 2010, pp. 1-5. DOI: 10.1109/GLOCOM.2010.5683485

[41] M. Doniec, M. Angermann, and D. Rus, "An End-to-End Signal Strength Model for Underwater Optical Communications," IEEE Journal of Oceanic Engineering, vol. 38, no. 4, pp. 743-757, Oct. 2013. DOI: 10.1109/JOE.2013.2278932

[42] S. Tang, Y. Dong, and X. Zhang, "Impulse Response Modeling for Underwater Wireless Optical Communication Links," IEEE Trans. on Communications, vol. 62, no. 1, pp. 226-234, Jan. 2014. DOI: 10.1109/TCOMM.2013.120713.130199

[43] D. Piao and G. Lu, "Application of Cooperative MIMO in the Ad Hoc Shallow Water Acoustic Sensor Network,". in IEEE 4th International Conference on Wireless Communications, Networking and Mobile Computing, WiCOM'08, pp. 14, Oct. 2008. DOI: 10.1109/WiCom.2008.980

[44] B. Gulbahar and O. B. Akan, "A communication theoretical modeling and analysis of underwater magneto-inductive wireless channels," IEEE Transactions on Wireless Communications, vol. 11, no. 9, pp. 3326-3334, 2012. DOI: 10.1109/TWC.2012.070912.111943

[45] R. J. Urick, Ambient Noise in the Sea. Peninsula Pub, 1986.

[46] M. Stojanovic, "On the relationship between capacity and distance in an underwater acoustic communication channel," ACM SIGMOBILE Mobile Comput. and Comm. Review (M2CR), vol. 11, pp. 34-43, Oct. 2007. DOI: 10.1145/1161039.1161049

[47] Z. Sun and I. Akyildiz, "Magnetic induction communications for wireless underground sensor networks," IEEE Trans. Antennas Propag., vol. 58, no. 7, pp. 2426-2435, 2010. DOI: 10.1109/TAP.2010.2048858

[48] S. Cui, A. J. Goldsmith, and A. Bahai, "Energy-Efficiency of MIMO and Cooperative MIMO Techniques in Sensor Networks," IEEE J. Sel. Areas Commun., vol. 22, no. 6, pp. 1089-1098, Aug. 2004. DOI: 10.1109/JSAC.2004.830916

[49] S. Cui, A. J. Goldsmith, and A. Bahai, "Energy-constrained modulation optimization," IEEE Trans.Wireless Commun., vol. 4, no. 5, pp. 2349-2360, Sep. 2005. DOI: 10.1109/TWC.2005.853882 
[50] M. Steyaert, B. De Muer, P. Leroux, M. Borremans, and K. Mertens, "Lowvoltage low-power CMOS-RF transceiver design," IEEE Trans. Microwave Theory Tech., vol. 50, pp. 281-287, Jan. 2002. DOI: 10.1109/22.981281

[51] T. H. Lee, The Design of CMOS Radio-Frequency Integrated Circuits, Cambridge, U.K.: Cambridge Univ. Press, 1998.

[52] A. G. Lazaropoulos and P. G. Cottis, "Capacity of overhead medium voltage power line communication channels," IEEE Trans. Power Del., vol. 25, no. 2, pp. 723-733, Apr. 2010. DOI: 10.1109/TPWRD.2009.2034907

[53] A. G. Lazaropoulos and P. G. Cottis, "Broadband transmission via underground medium-voltage power lines-Part II: capacity," IEEE Trans. Power Del., vol. 25, no. 4, pp. 2425-2434, Oct. 2010. DOI: 10.1109/TPWRD.2010.2052113

[54] A. Goldsmith, S. A. Jafar, N. Jindal, and S.Vishwanath, "Capacity limits of MIMO channels," IEEE J. Sel. Areas. Commun., vol. 21, no. 5, pp. 684-702, Jun. 2003. DOI: 10.1109/JSAC.2003.810294

[55] A. Schwager, D. Schneider, W. Bäschlin, A. Dilly, and J. Speidel, "MIMO PLC: Theory, Measurements and System Setup," in Proc. IEEE Int. Symp. Power Line Communications and Its Applications, Udine, Italy, Apr. 2011, pp. 48-53. DOI: 10.1109/ISPLC.2011.5764447

[56] B. Li, J. Huang, S. Zhou, K. Ball, M. Stojanovic, L. Freitag, and P. Willett, "MIMO-OFDM for high rate underwater acoustic communications," IEEE J. Ocean. Eng., vol. 34, no. 4, pp. 634-644, 2009. DOI: 10.1109/JOE.2009.2032005

[57] C. Polprasert, J. A. Ritcey, and M. Stojanovic, "Capacity of OFDM systems over fading underwater acoustic channels," IEEE Journal of Oceanic Engineering, vol. 36, no .4, pp. 514-524, 2011. DOI: 10.1109/JOE.2011.2167071

[58] X. Junfeng, K. Li, and G. Min, "Reliable and energy-efficient multipath communications in underwater sensor networks," IEEE Transactions on Parallel and Distributed Systems, vol. 23, no. 7, pp. 1326-1335, 2012. DOI: 10.1109/TPDS.2011.266

[59] C. Xiong, G. Y. Li, S. Zhang, Y. Chen, and S. Xu, "Energy- and spectralefficiency tradeoff in downlink OFDMA networks," IEEE Trans. Wireless Commun., vol. 10, no. 11, pp. 3874-3886, Nov. 2011. DOI: 10.1109/TWC.2011.091411.110249

[60] L. Zheng and D. N. C. Tse, "Diversity and multiplexing: A fundamental tradeoff in multiple-antenna channels," IEEE Trans. on Information Theory, vol. 49, no. 5, pp. 1073-1096, 2003. DOI: 10.1109/TIT.2003.810646

[61] C. Isheden and G. P. Fettweis, "Energy-efficient multi-carrier link adaptation with sum rate-dependent circuit power," in Proc. IEEE Global Telecommunications Conference, Miami, FL, USA, Dec. 2010, pp. 1-6. DOI: 10.1109/GLOCOM.2010.5683700

[62] C. Liu, Y. V. Zakharov, and T. Chen, "Doubly selective underwater acoustic channel model for a moving transmitter/receiver," IEEE Transactions on Vehicular Technology, vol. 61, no. 3, pp. 938-950, 2012. DOI: 10.1109/TVT.2012.2187226

[63] M. Stojanovic, "Design and Capacity Analysis of Cellular-Type Underwater Acoustic Networks,” IEEE J. Ocean. Eng., vol. 33, pp. 171-181, Apr. 2008. DOI: 10.1109/JOE.2008.920210

[64] C. H. Mar and W. K. G. Seah, "DS/CDMA throughput of a multi - hop sensor network in a Rayleigh fading underwater acoustic channel," Concurrency and 
Computation: Practice and Experience, vol. 19, no. 8, pp. 1129-1140, 2007. DOI: 10.1002/cpe.1163

[65] H. Kulhandjian, T. Melodia, and D. Koutsonikolas, "Securing underwater acoustic communications through analog network coding," In 2014 Eleventh Annual IEEE International Conference on Sensing, Communication, and Networking (SECON), pp. 266-274, 2014. DOI: 10.1109/SAHCN.2014.6990362

[66] H. Kulhandjian, T. Melodia, and D. Koutsonikolas, "CDMA-based analog network coding through interference cancellation for underwater acoustic sensor networks," In Proceedings of the Seventh ACM International Conference on Underwater Networks and Systems, p. 7, Nov. 2012.

[67] A. G. Kanatas, "Beamspace MIMO and Degrees of Freedom," In Parasitic Antenna Arrays for Wireless MIMO Systems, Springer New York, pp. 45-84, 2014. DOI: 10.1007/978-1-4614-7999-4_3

[68] P. S. Bithas, G. P. Efthymoglou, and A. G. Kanatas, "SEP of rectangular QAM in composite fading channels," AEU-International Journal of Electronics and Communications, vol. 69, no. 1, pp. 246-252, 2015. DOI: 10.1016/j.aeue.2014.09.011

[69] B. Han, V. I. Barousis, A. Kalis, C. B. Papadias, A. G. Kanatas, and R. Prasad, "A Single RF MIMO Loading Network for High-Order Modulation Schemes," International Journal of Antennas and Propagation, 2014.

[70] A. G. Kanatas, D. Vouyioukas, G. Zheng, and L. Clavier, "Beamforming Techniques for Wireless MIMO Relay Networks," International Journal of Antennas and Propagation, 2014.

[71] A. G. Lazaropoulos and P. Lazaropoulos, "Financially Stimulating Local Economies by Exploiting Communities' Microgrids: Power Trading and Hybrid Techno-Economic (HTE) Model," Trends in Renewable Energy, vol. 1, no. 3, pp. 131-184, Sep. 2015. DOI: 10.17737/tre.2015.1.3.0014

[72] A. G. Lazaropoulos, "Policies for Carbon Energy Footprint Reduction of Overhead Multiple-Input Multiple-Output High Voltage Broadband over Power Lines Networks," Trends in Renewable Energy, vol. 1, no. 2, pp. 87-118, Jun. 2015. DOI: 10.17737/tre.2015.1.2.0011

[73] A. G. Lazaropoulos, "Designing Broadband over Power Lines Networks Using the Techno-Economic Pedagogical (TEP) Method - Part I: Overhead High Voltage Networks and Their Capacity Characteristics," Trends in Renewable Energy, vol. 1, no. 1, pp. 16-42, Mar. 2015. DOI: 10.17737/tre.2015.1.1.002

[74] A. G. Lazaropoulos, "Designing Broadband over Power Lines Networks Using the Techno-Economic Pedagogical (TEP) Method - Part II: Overhead Low-Voltage and Medium-Voltage Channels and Their Modal Transmission Characteristics," Trends in Renewable Energy, vol. 1, no. 2, pp. 59-86, Jun. 2015. DOI: $10.17737 /$ tre.2015.1.2.006

Article copyright: (C) 2016 Athanasios G. Lazaropoulos. This is an open access article distributed under the terms of the Creative Commons Attribution 4.0 International License, which permits unrestricted use and distribution provided the original author and source are credited. 